\title{
Haemophilia A management with emicizumab: A survey of haematologists in the United States
}

Anisha M. Patel, Wendy E. Owens, Kathleen Poulos, Karina Raimundo, Gabriel Wong, Janet Bernard, Janet Powers, Richard H. Ko

Background: Emicizumab is a bispecific monoclonal antibody approved in the United States (US) for the treatment of people with haemophilia $\mathrm{A}(\mathrm{PwHA})$ with or without factor VIII (FVIII) inhibitors. Changes to haematologists' practices since the approval of emicizumab are of interest to the haemophilia $A$ community. Aim: To identify the clinical characteristics of PwHA receiving emicizumab (PwHArE) in the real-world setting and gain insight into the disease management practices of haematologists treating

ANISHA M. PATEL

Genentech, Inc., South San Francisco, CA, USA. Email:

patel.anisha@gene.com

WENDY E. OWENS

Genentech, Inc., South San Francisco, CA, USA

KATHLEEN POULOS

MedPanel, Waltham, MA, USA ; New affiliation: Ferrum

Health, Inc., San Francisco, CA, USA

KARINA RAIMUNDO

Genentech, Inc., South San Francisco, CA, USA

GABRIEL WONG

Genentech, Inc., South San Francisco, CA, USA; New

affiliation: Seagen Inc., San Francisco, CA, USA

JANET BERNARD

MedPanel, Waltham, MA, USA

JANET POWERS

MedPanel, Waltham, MA, USA

RICHARD H. KO

Genentech, Inc., South San Francisco, CA, USA

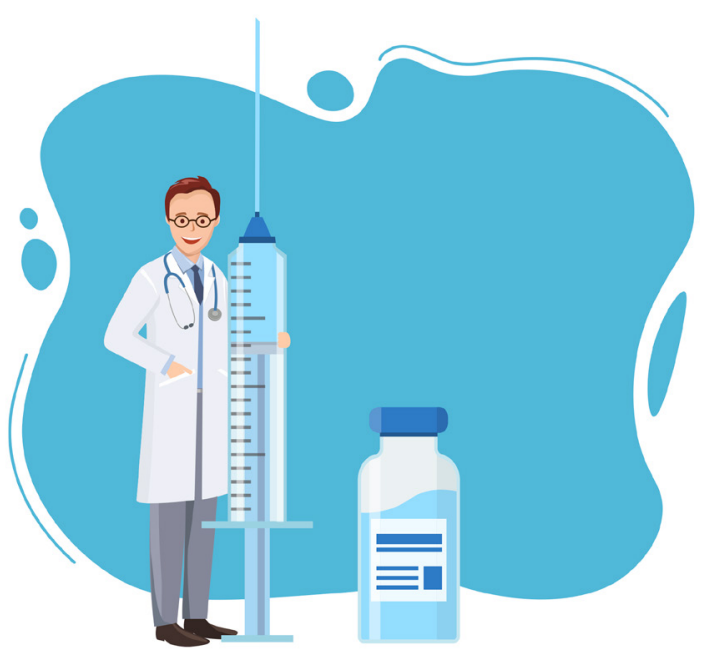

The introduction of emicizumab represents a paradigm shift in the treatment landscape for haemophilia A. Against this background, understanding how haematologists are adapting their management of treatment of people with haemophilia A receiving emicizumab is key.

PwHArE. Methods: In total, 50 haematologists across the US completed a one-time, 30-minute, online, qualitative survey consisting of 55 questions (including 11 screening questions) in May 2019. Haematologists were required to be board-certified in haematology, practising in the US, $\geq 2$ years postresidency experience, and currently treating $\geq 3$ PwHA with emicizumab. Results: Haematologists reported their PwHArE were mostly adults (aged $\geq 18$ years; $66 \%$ ) with severe phenotypes (66\%), with and 
without FVIII inhibitors. Haematologists perceived that PwHArE had similar or better treatment adherence (40\% and $50 \%$, respectively) compared with $\mathrm{PwHA}$ on other treatments, sought the same or lower levels of routine care $(72 \%$ and $14 \%$, respectively), and were similarly or more physically active $(52 \%$ and $32 \%$, respectively). Additionally, most haematologists currently using immune tolerance induction (ITI) in PwHArE reported using lower doses of FVIII (73\%) and shorter durations (45\%) for ITI. Conclusions: Availability of emicizumab has resulted in changes in the care of PwHArE, including bleed management, FVIII monitoring, activity guidance, surgery, and use of ITI. Understanding patterns of disease management can inform clinical care.

Keywords: emicizumab, haemophilia A, physicians, surveys and questionnaires

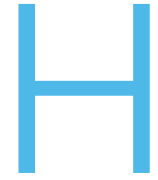

aemophilia $A$ is a congenital bleeding disorder associated with spontaneous or traumatic bleeding, most commonly into joints, muscles, and soft tissues ${ }^{[1]}$. It is caused

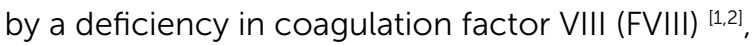
and can lead to secondary complications such as arthropathy ${ }^{[3]}$ and functional deficits ${ }^{[4-6]}$.

Prophylaxis with plasma-derived and recombinant factor concentrates has been used for haemophilia $A$ treatment for decades [7]; however, factor replacement therapies require regular intravenous infusions 2-3 times per week ${ }^{[8]}$. These burdensome treatment regimens can result in reduced adherence ${ }^{[9]}$. Furthermore, up to $20 \%$ of people with haemophilia A (PwHA) develop FVIII inhibitors, making them resistant to factor replacement therapies ${ }^{[10]}$. PwHA with FVIII inhibitors are typically treated with bypassing agents (BPAs) ${ }^{[11]}$. Some PwHA with FVIII inhibitors undertake immune tolerance induction (ITI), a costly and demanding process consisting of prolonged daily or alternate day high-dose FVIII intravenous administration with the aim of eradicating FVIII inhibitors ${ }^{[12,13]}$

Emicizumab, a recombinant, humanised, bispecific monoclonal antibody, is currently the only non-factor therapy approved for routine prophylaxis in $\mathrm{PwHA}$ with and without FVIII inhibitors. Emicizumab bridges activated FIX and FX to restore the function of missing activated FVIII in PWHA ${ }^{[14]}$, and is administered subcutaneously either once weekly, every 2 weeks, or every 4 weeks ${ }^{[15-17]}$. The pivotal phase 3 HAVEN clinical trial programme of emicizumab demonstrated its efficacy and safety; marked reductions in annualised bleeding rates of treated bleeds were observed, regardless of FVIII inhibitor status, and a majority of PwHA participating in the trials reported zero treated bleeds ${ }^{[15-18]}$. Data from HAVEN 1-4 ${ }^{[15-18]}$ supported the approval of emicizumab by the US Food and Drug Administration for routine prophylaxis in PWHA of all ages, regardless of inhibitor status ${ }^{[19]}$

As emicizumab has a different mechanism of action and mode of administration compared with factor replacement therapy or BPAs ${ }^{[20]}$, this raises the question of whether haematologists have adapted their care of PwHA accordingly. Many PwHA receiving emicizumab (PwHArE) experience few bleeds ${ }^{[21]}$, and the sustained therapeutic plasma levels of emicizumab ${ }^{[22]}$ offer different coverage for bleed prevention compared with the peaks and troughs of factor replacement. This may impact management of PwHArE by haematologists, including considerations such as surgery management and physical activity guidance. Use of emicizumab with ITI in the real-world setting has been covered through an increasing number of case reports ${ }^{[23]}$ and a retrospective review study ${ }^{[24]}$ on the concomitant use of emicizumab and ITI. As emicizumab prophylaxis is effective in preventing bleeding, FVIII can be administered solely for the purpose of inducing tolerance, and therefore at a lower dose or lower frequency than is typically used when undertaking ITI [13].

Due to these distinct features and the growing adoption of emicizumab in clinical practice, it is theorised that haematologists may adapt the way they treat PwHArE. The aim of this qualitative survey of haematologists was to understand the disease management practices of haematologists when managing and treating PwHArE, and to examine any changes in such practices.

\section{METHODS}

\section{Survey development}

A multi-level approach was used to develop the survey (Appendix / Supplementary Figure 1). To identify haematologists both to help with development of the survey and participate in the survey, the directory of the Centers for Disease Control and Prevention was used to locate haemophilia treatment centres (HTCs) in the US (https://dbdgateway.cdc.gov/HTCDirSearch.aspx), as well as using the proprietary panel of haematologists at MedPanel. Initial screening questions and a 60-minute in depth interview were conducted with five US-based 


\begin{tabular}{l|l|l} 
SURVEY & TOPIC & \multicolumn{1}{c}{$\begin{array}{c}\text { NUMBER OF } \\
\text { QUESTIONS }\end{array}$} \\
$\begin{array}{l}\text { Screening } \\
\text { questions }\end{array}$ & $\begin{array}{l}\text { Professional qualifications, medical specialty, board certification, post- } \\
\text { residency experience, practice setting, geographical location, number of } \\
\text { PWHA treated, and treatment }\end{array}$ & 11 \\
\hline Survey questions & & 9 \\
\hline Part 1 & Initiating treatment with emicizumab & 6 \\
\hline Part 2 & Bleed management & 2 \\
\hline Part 3 & Missed dose or discontinuation & 4 \\
\hline Part 4 & Monitoring & 4 \\
\hline Part 5 & Activity guidance & 2 \\
\hline Part 6 & Surgery management & 6 \\
\hline Part 7 & ITI & 8 \\
\hline Part 8 & Resource use and care & 1 \\
\hline Part 9 & Treatment access & 3 \\
\hline Part 10 & Change in overall disease management & 3 \\
\hline
\end{tabular}

ITI: immune tolerance induction; PwHA: people with haemophilia A

haematologists via telephone (with online screen sharing) to inform development of the survey questions. The haematologists included a mix of adult and paediatric treaters from both haemophilia treatment centre (HTC) and non-HTC settings who had experience treating PwHArE. The interviews were recorded and transcripts analysed to enable responses to be drafted into the pilot survey; following which, interviews with two other US haematologists were conducted via telephone to review the questions. Responses collected during the pilot survey were then used to finalise the one-time, 30-minute, online version of the survey. The survey was blinded: none of the haematologists involved in the development of the survey or those who completed the survey were identified by the authors of the manuscript at the time of the survey.

\section{Survey}

Invites for the survey were sent to haematologists across 140 HTCs as well as those working outside HTCs who were identified by MedPanel. From 8 May 2019 to 20 May 2019, eligible board-certified US paediatric and adolescent/adult haematologists were surveyed to understand their experience treating PwHArE, and to assess any changes in disease management practices in response to emicizumab. The haematologists surveyed during this time did not include any of the haematologists who had participated in the survey development and pilot survey. Participants were screened to determine if they met the inclusion criteria (Appendix / Supplementary Table 1), which included: board certification in haematology, practising in the US, $\geq 2$ years of post-residency experience, currently treating $\geq 3$ PwHArE. The survey topics ranged from emicizumab initiation to monitoring and special circumstances, such as surgery and ITI (Table 1). The final screening and survey questions are provided in the Appendix / Supplementary Table 3. Reported percentages are averaged across all survey responses.

\section{RESULTS}

A total of 208 haematologists responded to the invite. Fifty-one met the screening criteria and completed the survey ( $n=83$ screened out on one or more exclusion criteria; $n=74$ partially completed the screening or survey but did not finish). One of the completed surveys was excluded, leaving 50 surveys completed by 50 haematologists across 22 states (Appendix / Supplementary Table 2).

Of the 50 haematologists surveyed, more than three quarters $(78 \%)$ had $\geq 10$ years of post-residency experience and $46 \%$ practised at academic/teaching hospitals; of these, $78 \%(n=18 / 23)$ were federally funded. The majority (62\%) of haematologists treated $\geq 25$ PwHA per month; approximately $70 \%$ of all PwHA treated were adolescents/adults, and roughly one quarter (adult, 24.5\%; paediatric, 22.1\%) had FVIII inhibitors. All results presented here are as reported by the 50 haematologists who completed the survey, and the patient characteristics, outcomes, and experiences reported reflect the haematologists' perception of these factors. 
Clinical characteristics of PwHArE as reported by surveyed haematologists

Of the PwHArE reported by the haematologists surveyed, $66 \%$ were adults ( $\geq 18$ years old); $13 \%, 21 \%$, and $66 \%$ had mild, moderate, and severe haemophilia A, respectively (Table 2 ). When starting emicizumab, approximately $10 \%$ were previously untreated with FVIII. A majority of the PWHArE (64\%) engaged in moderate or high impact physical activities. When asked "What are the five most important patient characteristics that you considered when initiating treatment with emicizumab?", the most common options chosen by the surveyed haematologists were: presence of FVIII inhibitors (70\%), high treatment burden (62\%), and frequency of bleeds (60\%).

\section{Initiating treatment with emicizumab}

In total, $86 \%$ of surveyed haematologists reported that, in most cases, they initiated the discussion concerning emicizumab prophylaxis, with the remaining $14 \%$ reporting that, in most cases, the $\mathrm{PwHA}$ (or their caregiver) initiated the conversation. When providing reasons for initiating this discussion, haematologists could select multiple answers; lowering treatment burden (64\%), improving quality of life (QoL; 64\%), FVIII inhibitor development (56\%), improving bleed management (56\%), and venous access issues (48\%) were the top five reasons reported (Figure 1).

\section{Bleed management}

Haematologists could similarly select multiple answers concerning bleed management. To assist PwHArE (and their caregivers) in recognising breakthrough bleeds, some haematologists provided educational materials (68\%) or suggested PwHArE call their healthcare provider (HCP) for diagnosis support and to discuss bleed symptoms (70\%), while others reported that their PwHArE and caregivers know how to identify current bleeds (64\%). Other means, such as providing an ultrasound scan, were offered infrequently (16\%).

About one third (34\%) of haematologists surveyed reported that they have changed their guidance on bleed treatment for PwHArE. Changes to guidance stratified by FVIII inhibitor status are shown in Figure 2; haematologists responded that they are more likely to advise PwHArE to call an HCP to discuss symptoms and treatment, regardless of FVIII inhibitor status. When asked what percentage of time PwHArE seek medical care for minor and significant bleeds, haematologists reported that PwHArE with FVIII inhibitors sought slightly more care than those without
Table 2. Demographics and clinical characteristics of PwHArE treated by haematologists in this survey, as reported by haematologists.

These data are presented as perceived by the haematologists who completed the survey and do not include patient-level data

\begin{tabular}{|c|c|}
\hline SURVEY & $\begin{array}{l}\text { PROPORTION OF } \\
\text { PWHArE TREATED BY } \\
\text { THE HAEMATOLOGISTS }\end{array}$ \\
\hline \multicolumn{2}{|l|}{ PwHA age groups } \\
\hline Infants (aged 0-12 months) & 1.6 \\
\hline Children (aged 1-11 years) & 16.2 \\
\hline Adolescents (aged $12-17$ years) & 16.2 \\
\hline Adults (aged $18-65$ years) & 55.3 \\
\hline Older adults (aged $>65$ years) & 10.6 \\
\hline PUPs* & 10.1 \\
\hline \multicolumn{2}{|l|}{ Disease severity $^{\dagger}$} \\
\hline Mild & 12.8 \\
\hline Moderate & 21.3 \\
\hline Severe & 65.9 \\
\hline
\end{tabular}

Frequency of bleeds while receiving emici-zumab (mild disease) ${ }^{\dagger}$

\begin{tabular}{l|l}
$\leq 2$ bleeds/year & $64.3^{\ddagger}$ \\
$>2$ bleeds/year & $35.7^{\ddagger}$
\end{tabular}

\begin{tabular}{|c|c|}
\hline \multicolumn{2}{|c|}{$\begin{array}{l}\text { Frequency of bleeds while receiving emici-zumab } \\
\text { (moderate disease) }\end{array}$} \\
\hline$\leq 2$ bleeds/year & $54.0^{\ddagger}$ \\
\hline$>2$ bleeds/year & $46.0^{\ddagger}$ \\
\hline
\end{tabular}

\section{Current FVIII inhibitors}

\begin{tabular}{l|l} 
Low titre inhibitors & 35.3 \\
\hline High titre inhibitors & 64.7
\end{tabular}

\begin{tabular}{l|l|}
\hline \multicolumn{2}{l}{ Previous FVIII inhibitors that have resolved } \\
\hline Low titre inhibitors & 39.7 \\
\hline High titre inhibitors & 60.3 \\
\hline Physical activity level & 24.0 \\
\hline Low impact activities & 34.5 \\
\hline Moderate impact activities & 29.7 \\
\hline High impact activities & 11.8 \\
\hline $\begin{array}{l}\text { Typically fit into }>1 \text { category of } \\
\text { physical activity }\end{array}$ & \\
\hline
\end{tabular}

FVIII: factor VIII; PUP: previously untreated person with haemophilia $A$; PwHA: people with haemophilia $A$; PwHArE: people with haemophilia $A$ receiving emicizumab

* Not previously treated with FVIII

† As determined by FVIII genotype. Frequency of bleeds were reported for people with mild and moderate haemophilia A only ‡ Only haematologists who indicated they treated mild $(n=22)$ or moderate $(n=37)$ PwHArE, respectively, were eligible to respond to this question

If PwHA participating in mixed activities 
Figure 1. Reasons for PwHA starting emicizumab treatment, as perceived by haematologists $(\mathrm{N}=50)$

These reasons are presented as perceived by the haematologists who completed the survey and do not include patient-reported data

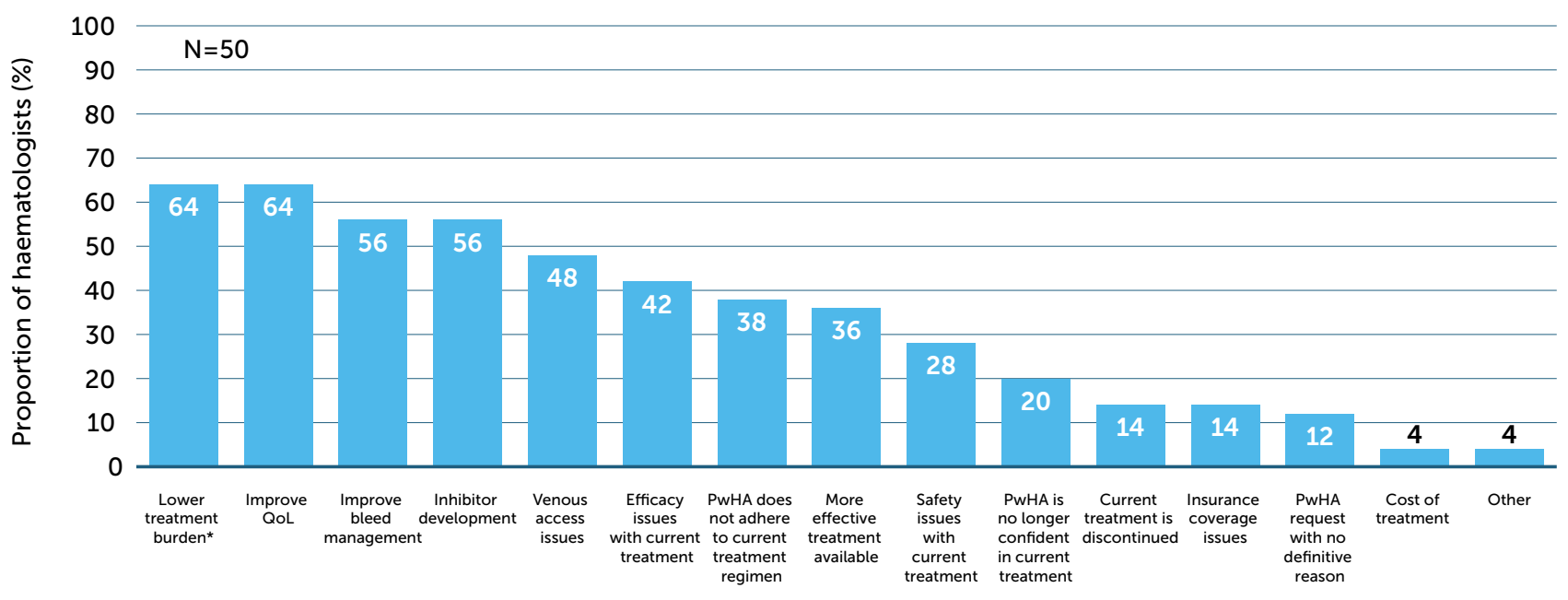

Reasons for switching to or initiating emicizumab treatment

PwHA: person/people with haemophilia A; QoL: quality of life

* Examples: ease of administration and lower administration frequency

FVIII inhibitors (minor bleeds: with FVIII inhibitors, 41\%; without FVIII inhibitors, 39\%; significant bleeds: with FVIII inhibitors, 67\%; without FVIII inhibitors, 60\%); however, the numerical difference between subgroups was minimal.

Treatment guidance varied by FVIII inhibitor status and bleed type (Appendix / Supplementary Figure 2), but overall, a large proportion of haematologists recommended a few strategies, including asking PwHArE to treat as soon as a joint, muscle, or other internal bleed is suspected, regardless of FVIII inhibitor status. For superficial/other soft tissue bleeds, 50\% of haematologists recommended that PwHArE with FVIII inhibitors call an HCP to discuss symptoms and obtain treatment advice before treating, while 54\% recommended that those without FVIII inhibitors take a single dose of FVIII as soon as a bleed is suspected. A majority of haematologists (48\%) advised the same number of FVIII/BPA doses should be kept on hand by PwHArE compared with those on other HA treatment (Appendix / Supplementary Figure 3a); for most haematologists (42\%), this recommendation was 3-4 FVIII/BPA doses (Appendix / Supplementary Figure 3b).

\section{Adherence, missed dose, and discontinuation}

When asked "How does the patient's level of adherence with emicizumab compare to your HA patients on other treatments?", 50\% of haematologists surveyed perceived that PwHArE have better or significantly better adherence to their prophylactic regimen, while 40\% perceived a similar adherence, compared with $\mathrm{PwHA}$ taking other prophylaxis. Most haematologists reported monitoring adherence through assessment at each office visit (74\%) and working with a multidisciplinary care team to track adherence (44\%). To encourage treatment adherence, $86 \%$ of haematologists reported discussing its importance with PwHArE at each office visit (Appendix / Supplementary Figure 3c).

Overall, $94 \%$ of haematologists reported that they provided guidance to PWHA on a missed emicizumab dose. Administering the missed dose as soon as possible and then resuming the recommended dosing schedule was the most frequently reported advice (44\%), followed by administering the next dose as planned (26\%) and administering the missed dose as soon as possible before contacting an HCP to discuss resuming the dosing schedule (24\%).

When queried about factors contributing to $\mathrm{PWHA}$ discontinuing emicizumab, insurance coverage issues (70\%), treatment cost (50\%), personal choice of the individual (44\%), and tolerability of subcutaneous injections (44\%) were the most frequently cited reasons.

\section{Monitoring}

Some haematologists reported less frequent measuring for FVIII activity (52\%) and testing for FVIII inhibitors (28\%) in PwHArE compared with PwHA taking other 
Figure 2. Changes to haematologists' $(n=17)^{*}$ guidance on bleed management in PwHA with FVIII inhibitors (a) and without FVIII inhibitors (b), since starting emicizumab

2a. PwHA with FVIII inhibitors

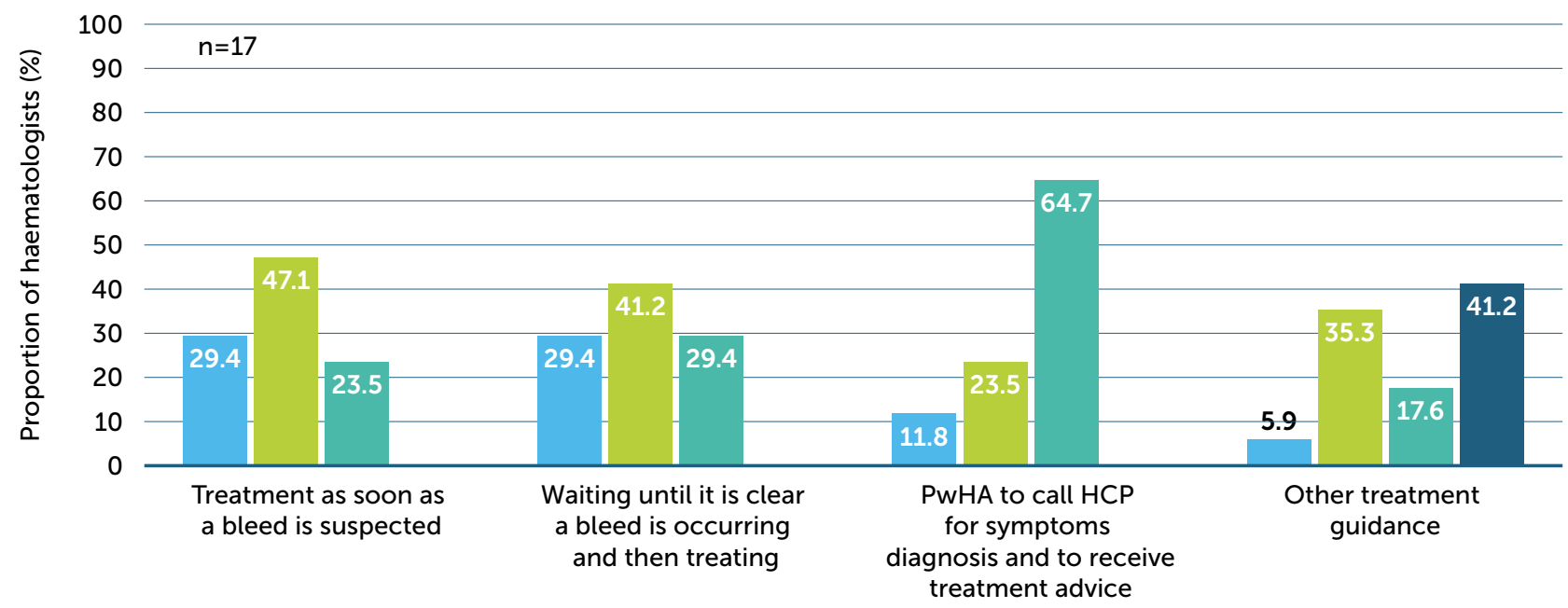

2b. PwHA without FVIII inhibitors



Less likely to recommend

More likely to recommend

Recommend about the same

Not sure / NA

FVIII: factor VIII; HCP: healthcare professional; NA: not applicable; PwHA: person/people with haemophilia A

* The remainder of the haematologists surveyed $(n=33 / 50)$ indicated that they did not change their bleed management guidance when their patients began receiving emicizumab

haemophilia A treatments, while 34\% and 54\% were measuring and testing about the same, respectively.

For PwHA with FVIII inhibitors, haematologists were more likely to report measuring FVIII activity monthly (18\%) and at additional times when they thought it was necessary (64\%) compared with PwHA without FVIII inhibitors ( $6 \%$ and $58 \%$, respectively). Others reported measuring FVIII activity for PwHA with and without FVIII inhibitors once a quarter $130 \%$ and $36 \%$, respectively), once a year (22\% and $26 \%$ ), and upon PwHA/caregiver request (both 30\%) (Appendix / Supplementary Figure 4). Most haematologists (76\%) also reported testing for FVIII inhibitors when they felt it was necessary. For PwHA without FVIII inhibitors, haematologists reported testing for inhibitors once per quarter or once per year (36\% each; Appendix / Supplementary Figure 4); however, the majority (60\%) reported that they would also test at additional times when they felt it was necessary.

\section{Activity guidance}

When asked "In general, how has the physical activity level changed in your patients after starting treatment with emicizumab?", the majority (84\%) of haematologists 
Figure 3. Haematologists' perceptions of (a) how physical activity levels have changed in PwHA since starting emicizumab ( $N=50$ ), (b) what type of physical activity guidance they provide to PwHArE $(n=27)^{*}$, and (c) if their guidance changes once PwHA start taking emicizumab $(n=27)^{*}$

These outcomes are presented as perceived by the haematologists who completed the survey and do not include patient-reported data

3a. Change in PwHA physical activity levels since starting emicizumab $(\mathrm{N}=50)$

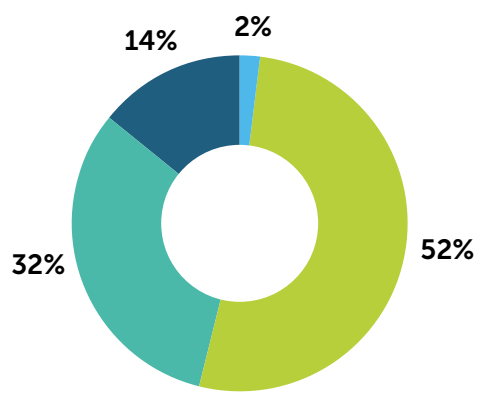

PwHA are less active

PwHA are more active

PwHA have similar activity levels

Not sure

FVIII: factor VIII; PwHA: person/people with haemophilia A; PwHArE: person/people with haemophilia A receiving emicizumab

* The remainder of the haematologists $(N=23 / 50)$ indicated that they did not provide guidance for recreational physical activities

3b. Type of physical activity guidance provided to $\operatorname{PwHArE}(n=27)$

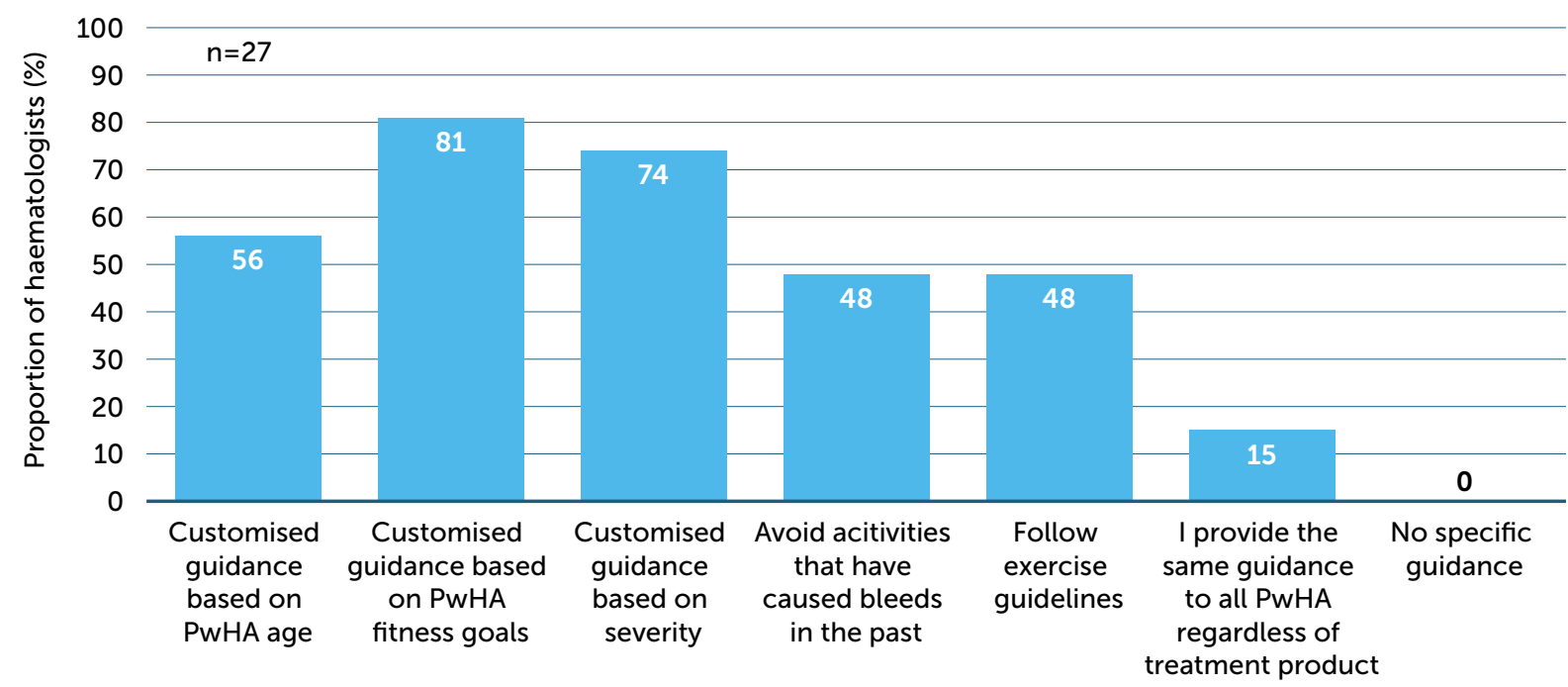

3c. Does guidance on physical activity change once PwHA start taking emicizumab $(n=27)$

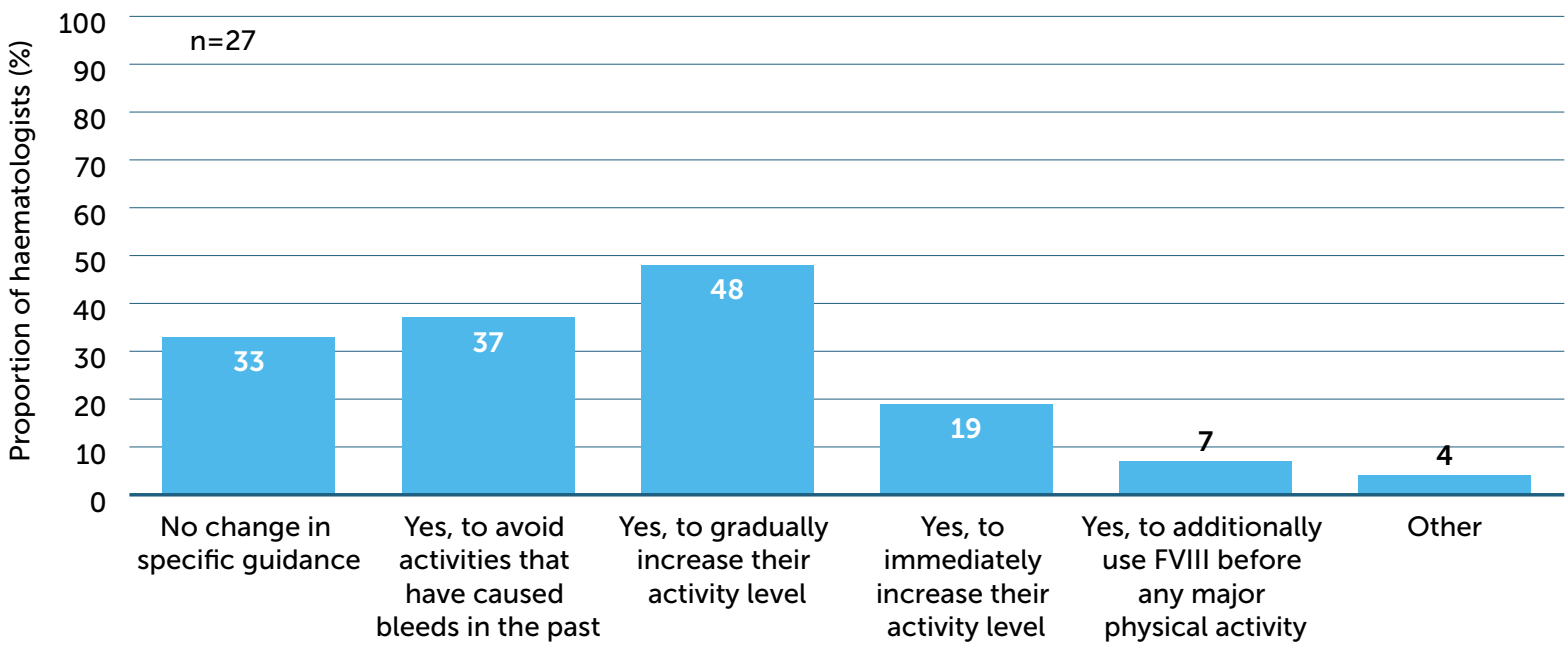


Figure 4. ITI products and bleed treatments used or considered for use in PwHArE $(n=30)$

4a. ITI products used* or considered for use ${ }^{\dagger}$ in PwHArE

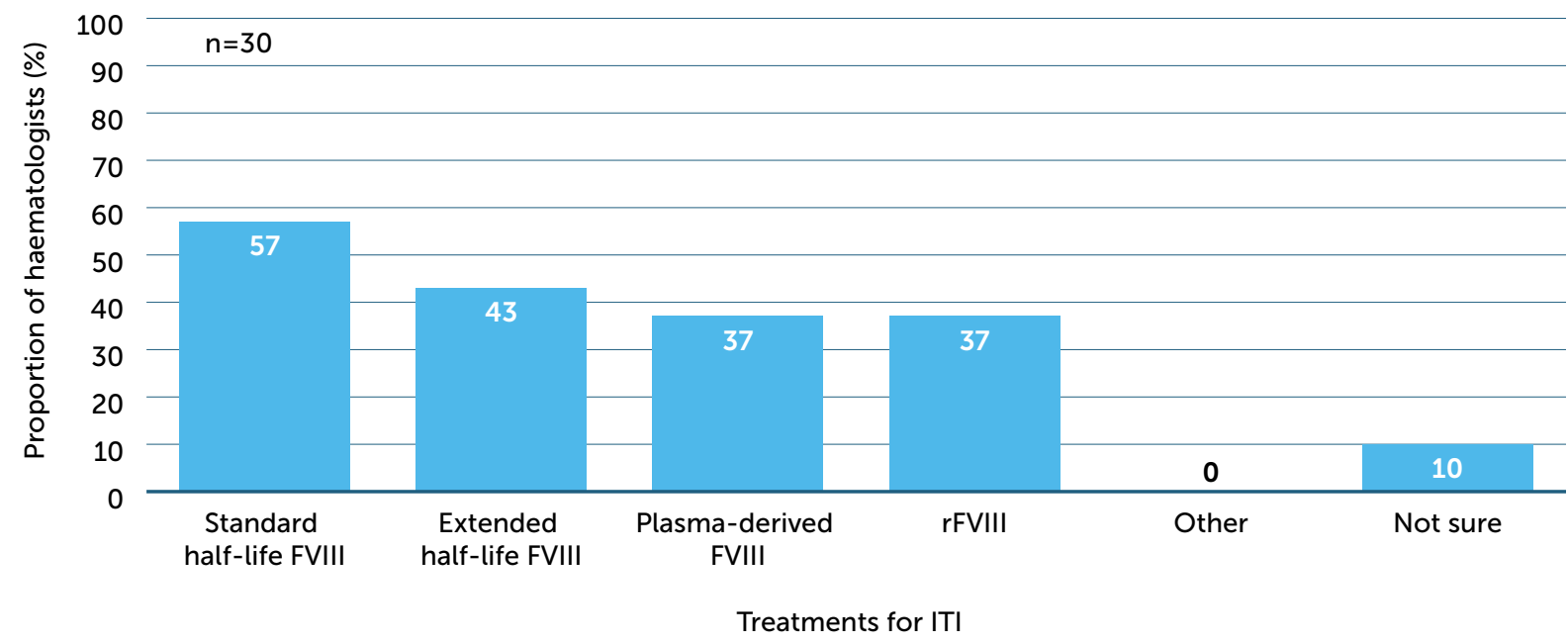

4b. Bleed treatments used or considered for use during ITI for PwHArE

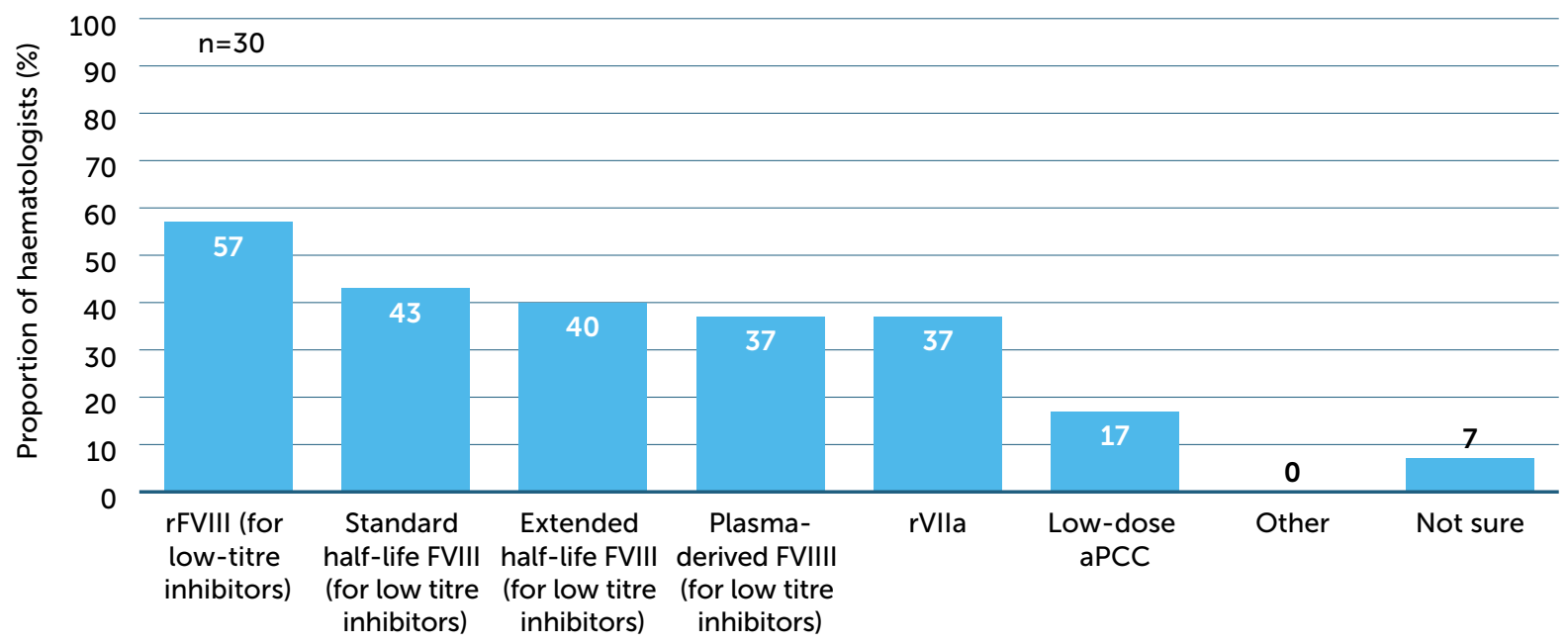

Treatments for breakthrough bleeds during ITI

aPCC: activated prothrombin complex concentrate; FVIII: factor VIII; ITI: immune tolerance induction; PwHArE: person/people with haemophilia A receiving emicizumab; rFVIII: recombinant factor VIII; rFVIla: recombinant factor VIIa

* PwHArE and receiving ITI: $n=11$

$\dagger$ PwHArE and who may receive ITI in the future: $n=19$

surveyed perceived that the recreational physical activity levels of $\mathrm{PwHA}$ remained the same (52\%) or increased (32\%) after starting emicizumab treatment (Figure 3a). Among the 27 haematologists who provided specific guidance on recreational physical activities, most developed customised plans for PwHArE based on fitness goals (81\%), disease severity (74\%), and age (56\%; Figure 3b); further, after starting emicizumab, $48 \%$ of haematologists who provide activity guidance recommended that PwHArE gradually increase these activities, while $37 \%$ also recommended to avoid activities that have caused bleeds in the past (Figure $3 c$ ).

\section{Surgery management}

Multiple strategies in the surgical management of PwHArE were reported, with 22\% $(n=11 / 50)$ of the haematologists surveyed treating PwHArE who have undergone surgery at the time of the survey. Haematologists reported varying levels of management, from close monitoring of bleed control to pre- or post-operatively using other haemostatic treatments, depending on the type of surgery and risk of bleeding Overall, the least preferred management option was suspending or stopping emicizumab prior to surgery. For minor and major surgeries with a high risk of 
bleeding, 91\% ( $n=10 / 11)$ of haematologists managed PwHArE pre operatively using other haemostatic treatments (BPAs or FVIII; Appendix / Supplementary Figure 5) for additional coverage.

\section{Immune tolerance induction}

A minority of haematologists surveyed $(22 \%, n=11)$ reported that they were carrying out ITI and 38\% ( $n=19)$ were considering using it in the future. Among those currently carrying out or considering initiating ITI in the future, treatment choice for ITI and bleeds in PWHArE varied (Figure $4 a$ and $4 b$, respectively).

Of the 11 haematologists currently carrying out ITI in PWHArE, the majority (73\%) opted to prescribe a lower dose of FVIII for ITI (e.g. FVIII 50-100 IU/kg 2 or 3 times per week) compared with $\mathrm{PwHA}$ taking other haemophilia A treatments. Almost half (45\%) opted to shorten the duration of ITI (e.g. $\leq 12$ months), with the next most popular option being no change to duration (36\%) (Appendix / Supplementary Figure 6a). Most haematologists (64\%) reported managing the timing of emicizumab dosing during ITI, with a minority (27\%) stating that no additional management was required.

Among the 19 haematologists who were considering ITI, approximately half (53\%) planned to prescribe a lower dose of FVIII for PwHArE compared with those taking other haemophilia A treatments. Around half $(47 \%)$ planned no change to the duration and about a third (32\%) planned to shorten the duration (Supplementary Figure 6b). Responses regarding how they intended to manage this treatment were mixed; $42 \%$ indicated that no additional management was planned; an equal amount ( $16 \%$ each) planned to either manage the timing of emicizumab dosing or suspend treatment with emicizumab; $5 \%$ indicated other methods; and $21 \%$ were unsure.

\section{Resource use and care}

In this survey, routine care was defined as annual visits and scheduled appointments. Most haematologists surveyed (72\%) perceived that PWHA seek the same amount of routine care pre- and post-emicizumab prophylaxis initiation, while $14 \%$ perceived that less routine care was sought. The proportion of haematologists who perceived that PwHArE sought less non routine care (trauma, major bleed, and emergency surgery) post-emicizumab initiation was $32 \% ; 46 \%$ of the haematologists perceived that nonroutine care levels remained the same. Follow-up care was defined as post-surgery and trauma care. Over half $(56 \%)$ of the haematologists surveyed perceived that PwHArE seek the same amount of follow-up care as they did prior to starting emicizumab prophylaxis (Figure 5a).

When comparing with $\mathrm{PWHA}$ receiving other haemophilia A treatment, $78 \%$ of haematologists perceived that PwHArE seek the same amount of routine care, while $14 \%$ perceived that they seek less. Regarding non-routine care, a higher proportion of haematologists (36\%) perceived that PwHArE sought care less frequently compared with $\mathrm{PwHA}$ receiving other haemophilia A treatment; $58 \%$ perceived these levels were about the same. The top five reasons PwHArE received non-routine care were: nontrauma-related breakthrough bleeds (76\%); traumarelated bleeds (54\%); guidance needed on FVIII or BPA use (52\%); follow-up care after hospitalisations/ emergency room visits (48\%); and participation in physically demanding work or school activities (44\%). After trauma or a surgery, most haematologists (60\%) thought that PwHArE sought similar levels of follow-up care compared with those taking other haemophilia $A$ treatments (Figure 5b).

\section{Treatment access}

When surveyed on the frequency of issues encountered with insurance coverage of emicizumab by health insurance providers, at the time of the survey, $46 \%$ of haematologists believed that the PwHA in their care frequently/always experience these issues with emicizumab. In addition, 38\% of haematologists believed that PwHArE frequently/always experience issues with FVIII/BPA coverage for bleed treatment and $22 \%$ found the same for care (e.g. office visits, physical therapy) while on emicizumab (Figure 6).

\section{Changes in overall disease management}

When asked how the level of disease management support they provided to PwHArE had changed since starting emicizumab, the majority (58\%) of haematologists reported no change compared with the support they offered to PwHA taking other haemophilia A treatments (Appendix / Supplementary Figure 7). Of those who reported that they provided less support $(n=6)$, they cited fewer bleeds $(100 \%)$, fewer disease-related issues versus $\mathrm{PwHA}$ taking other haemophilia A treatments (83\%), and not being as concerned about breakthrough bleeds for PwHArE $(67 \%)$ as the most common reasons. For those reporting that more support was required $(n=13)$, limited longterm efficacy and safety data (at the time of survey) was the most frequently cited reason (54\%). 
Figure 5. Haematologist-reported changes to (a) level of care sought by PwHA since starting emicizumab and (b) level of care sought by PwHArE compared with PwHA taking other haemophilia A treatments $(\mathrm{N}=50)$

These outcomes are presented as perceived by the haematologists who completed the survey and do not include patient-reported data

5a. Level of care sought by PwHA since starting emicizumab

Routine care



$72 \%$
Non-routine care

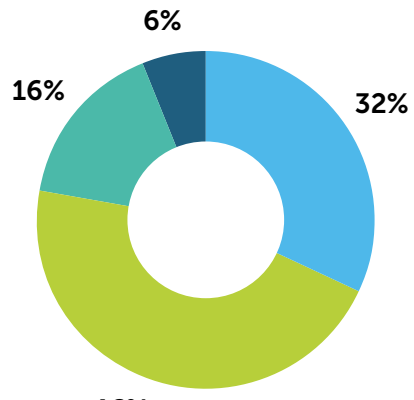

$46 \%$
Follow-up care

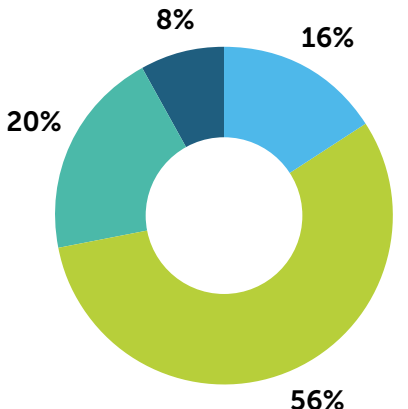

$56 \%$

5b. Level of care sought by PwHArE in comparison with PwHA taking other treatments

Routine care

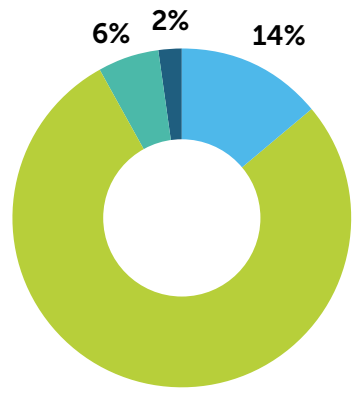

$78 \%$
Non-routine care

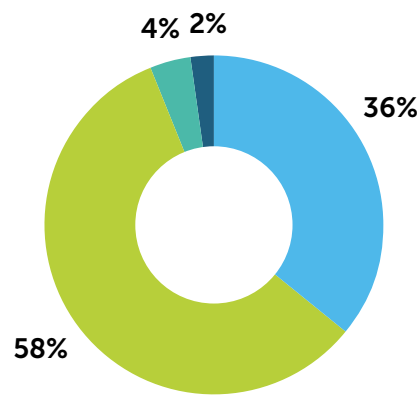

Same

$36 \%$

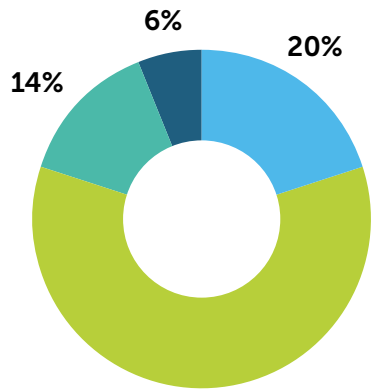

$60 \%$

Not sure

PwHA: person/people with haemophilia A; PwHArE: person/people with haemophilia A receiving emicizumab

Figure 6. Proportion of PwHA who experience insurance coverage issues for emicizumab treatment (a), FVIII/BPAs treatment for bleed management while taking emicizumab (b), and care for haemophilia A while taking emicizumab* (c), as reported by haematologists ( $\mathrm{N}=50$ ) These experiences are presented as perceived by the haematologists who completed the survey and do not include patient-reported data

\section{6a. Emicizumab}

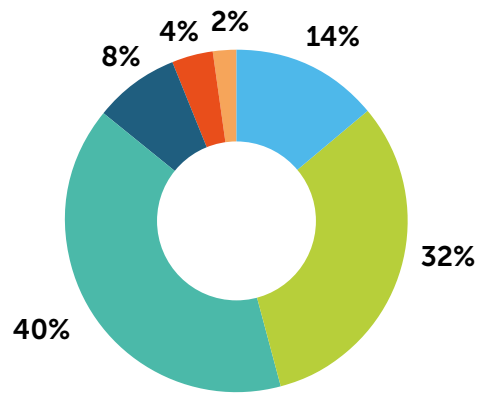

Always

Frequently 6b. FVIII/BPAs for bleed management

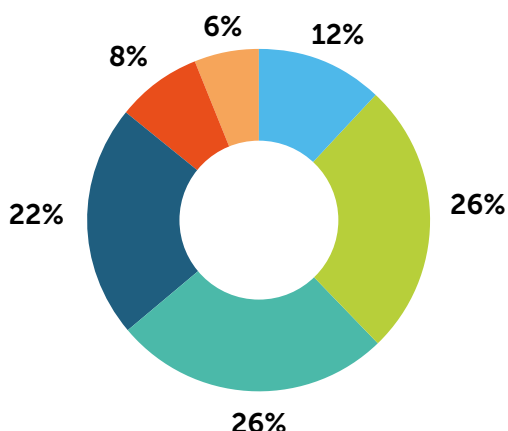

Occasionally

Rarely

BPA: bypassing agent; FVIII: factor VIII; HA: haemophilia A; PwHA: people with haemophilia A

*Example: office visits and physical therapy

6c. Haemophilia care

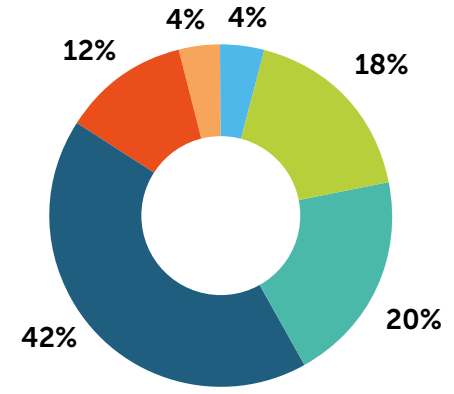

Never

Not sure 


\section{DISCUSSION}

A total of 50 haematologists in the US were included in this qualitative survey, most of whom practiced at federally funded haemophilia treatment centres. The majority of PwHA treated by these haematologists were adults aged 18 years or over, with severe haemophilia A. Approximately $10 \%$ of PWHA had not received FVIII treatment prior to starting emicizumab.

The most common reasons given for initiating emicizumab prophylaxis in PwHA included: lowering treatment burden, improving QoL, FVIII inhibitor development, improving bleed management, and venous access issues. This may be due to the reported efficacy of emicizumab in PwHA regardless of FVIII inhibitors ${ }^{[15,18]}$, and its subcutaneous administration on a weekly, every 2 weeks, or every 4 weeks basis ${ }^{[17]}$, which are afforded by its mechanism of action [20]. Emicizumab is not structurally analogous to FVIII ${ }^{[14]}$ and therefore can be used in PwHA with and without FVIII inhibitors without causing the development of new FVIII inhibitors ${ }^{[199}$. It was anticipated that this would be a strong motivating factor for switching to emicizumab, which is supported by the results of this survey. The reasons for initiating emicizumab chosen by haematologists in this survey are supported by data from the HAVEN clinical trials, including improvements in QoL, as quantified using measures such as the Haemophilia Quality of Life Questionnaire for Adults and the Haemophilia-specific Quality of Life Questionnaire for Children Short Form [25-27]; high rates of zero treated bleeds ${ }^{[21]}$; and the results of the Emicizumab Preference Survey ${ }^{[28]}$. It was also perceived by the haematologists surveyed that the majority of PwHA maintained or increased their recreational physical activity levels after starting emicizumab, which may play a role in improving their QoL.

Most haematologists surveyed reported no change in the disease management support sought by PwHArE since starting emicizumab, and that they receive a similar level of support compared with PwHA receiving other haemophilia A treatments. Around a quarter of haematologists surveyed reported that PwHArE sought more support; this may be due to the novelty of emicizumab, although this consideration likely only applies to the initial period after treatment switch. The primary reason given for this increased support was limited long-term efficacy and safety data at the time of the survey; however, it should be noted that more long-term data and real-world evidence have since been published ${ }^{[21,29-32]}$. Of the haematologists who reported that PwHArE sought less support than previously, and less than PwHA receiving other haemophilia A treatments, most indicated that this was due to fewer bleeds and disease-related issues, and less concern regarding breakthrough bleeding in PwHArE, an opinion supported by data from the HAVEN clinical trial programme ${ }^{[15-18]}$. There were some differences in disease management when PwHA began receiving emicizumab, as noted by the haematologists. For example, in the event of a bleed, they were more likely to advise PwHArE to call an HCP to discuss symptoms and treatment for guidance on determining whether a bleeding episode could be confirmed, which may reflect the need to confirm a bleed given how new emicizumab was at the time of the survey. Over half of the haematologists surveyed reported monitoring less frequently for FVIII activity for PwHArE compared with other haemophilia A treatments; this may be due to the difficulty of quantifying FVIII equivalence for a therapy with a fundamentally different mechanism of action [33], or it may reflect the sustained protection afforded ${ }^{[22]}$, in contrast to the peaks and troughs of FVIII replacement therapy.

Although only small numbers of haematologists in the survey had handled more challenging situations such as surgery and ITI, these are important topics in the haemophilia community. As such they were included in the survey to gather information on best practice and haematologists' experience of how they can be managed in PwHArE. Many haematologists administered FVIII or BPAs pre-, intra-, and postoperatively for PwHArE undergoing major and minor surgeries, more so if there was judged to be a higher bleed risk. These findings were aligned with the HAVEN programme, in which $83 \%$ of major surgeries were performed with prophylactic coagulation factor and the majority had no treated post-operative bleeds ${ }^{[34]}$. Most haematologists used lower doses of FVIII for ITI and would maintain or shorten duration. This is presumably due to the prophylactic bleed protection provided by emicizumab, so FVIII can be dispensed only at the dose needed to induce tolerance ${ }^{[13]}$.

Emicizumab is a bispecific humanised monoclonal antibody in a treatment landscape primarily characterised by iterative improvements on factor replacement. As it becomes more widely prescribed, the experience of haematologists currently using emicizumab to treat PwHA is invaluable. This survey can therefore be used to inform clinical practice and decision making by haematologists when initiating treatment with emicizumab in the PwHA under their 
care. Moreover, the implications of these findings can be used to guide insurance coverage policies; for example, the recommendation by many of the haematologists surveyed was for PwHA to keep 3-4 doses of FVIII or BPAs on hand to treat any potential breakthrough bleeds. Furthermore, the haematologists consulted in this survey collectively highlighted the treatment access issues encountered by $\mathrm{PwHA}$, which were cited as the most common reason for discontinuing emicizumab. This finding is consistent with data from a survey of people with haemophilia where insurer-restricted access to HTCs was highlighted as an issue of concern ${ }^{[35]}$. Emicizumab has an established long-term safety profile, proven efficacy in bleed prevention [21], and, as illustrated by better treatment adherence reported in this survey compared with other haemophilia A treatments, a low treatment burden. It has been found that high treatment adherence is vital for achieving optimal clinical outcomes and improvements in haemophilia-Arelated QoL ${ }^{[6,36,37] ;}$ therefore, it is critical that appropriate PwHA should be able to access this therapy.

Potential economic benefits of emicizumab, particularly in PwHA with FVIII inhibitors [38-40], were also expanded upon by the results of this survey. Despite the cost, ITI remains the only proven modality for eradicating FVIII inhibitors ${ }^{[41]}$. In the current survey, most haematologists indicated that they would treat PwHArE with a lower dose of FVIII for ITI and many also indicated that they would shorten the duration of ITI, representing potential cost savings to the insurance provider and/or PwHA. Two recent Institute of Clinical and Economic Review reports demonstrated cost savings of emicizumab over FVIII and BPA therapies in noninhibitor and inhibitor populations, respectively ${ }^{[42-43]}$. Emicizumab's cost-effectiveness has also been shown in other short- and long-term economic models [38-40].

\section{Limitations}

Limitations of the data include those inherent to survey research, such as restrictive wording of the questions and responses, subjective interpretations by respondents, and human errors in memory and reporting ${ }^{[44]}$. Further limitations are the relatively small sample size of haematologists surveyed $(\mathrm{N}=50)$ and limited number of years' experience managing PwHArE. Additionally, although it was noted that roughly one quarter of the PwHA managed by the haematologists in this survey had FVIII inhibitors (an important factor in the context of disease management), this was not quantified for the PwHArE. However, since
$56 \%$ of haematologists reported that FVIII inhibitor development was one of the most important reasons for initiating treatment with emicizumab prophylaxis, it is likely that a higher proportion of their PwHArE had FVIII inhibitors compared with the wider population of $\mathrm{PwHA}$ that they manage. The proportion of PwHArE with FVIII inhibitors may have impacted many of the survey findings, for example bleed frequency, physical activity, level of routine care, and treatment adherence. Furthermore, the findings reported here represent the haematologists' own personal experience and opinions and have not been confirmed as best practice in a trial setting; as such, reported changes in disease management practice should be interpreted with caution. Finally, the aspects of patient experience described here (for example, treatment adherence and treatment burden) are according to the surveyed haematologists' perception and not the patient's own report, and may therefore be subject to the haematologists' own bias. As the availability and quantity of post-approval emicizumab data and experience increases, new analyses will enable the generalisability of the results presented herein to be analysed and interpreted further.

\section{CONCLUSION}

The advent of emicizumab, a fully prophylactic treatment with subcutaneous administration once weekly, every 2 weeks, or every 4 weeks, represents a paradigm shift in the haemophilia A treatment landscape. Publishing the experience of haematologists using emicizumab in a real-world setting is important to inform clinical practice and disease management in PwHArE as its availability and use increases. The findings of this survey illustrate that some haematologists have adapted their management of haemophilia A in PwHArE, including how they handle bleed management, FVIII monitoring, and situations such as surgery and ITI. As emicizumab is increasingly prescribed and long-term, post-approval data continue to become available, patient characteristics, treatment methods, and disease management practice should continue to be monitored to support haematologists' treatment and care of $\mathrm{PwHA}$.

\section{AUTHOR CONTRIBUTIONS}

A. M. Patel, W. E. Owens, A. K. Poulos, J. Bernard and J. Powers carried out survey development and analysis of survey results. A. M. Patel, W. E. Owens, K. Raimundo, G. Wong and R. H. Ko contributed to the design and interpretation of the survey. All authors have reviewed 
and approved the manuscript prior to submission. All authors agree to be accountable for all aspects of the work.

\section{DECLARATION OF INTEREST}

None of the authors received honoraria or fees for their contribution to the development of this manuscript. A. M. Patel is a current employee of Genentech, Inc. and holds shares in F. Hoffmann-La Roche Ltd. W. E. Owens is a current employee of PRO Unlimited and has received consultancy from Genentech, Inc. via PRO Unlimited. K. Poulos is a previous employee of MedPanel, Inc. and Aquent, and is a current employee of Ferrum Health, Inc. K. Raimundo is a current employee of and holds shares in F. Hoffmann-La Roche, Ltd. G. Wong is a previous employee of Genentech, Inc. and a current employee of Seagen. J. Bernard and J. Powers are current employees of MedPanel, Inc. R. H. Ko is a current employee of Genentech, Inc. and holds shares in F. Hoffmann-La Roche, Ltd.

\section{DATA SHARING STATEMENT}

Qualified researchers may request access to individual patient-level data through the clinical study data request platform (https://vivli.org/). Further details on Roche's criteria for eligible studies are available here (https://vivli.org/members/ourmembers/). For further details on Roche's Global Policy on the Sharing of Clinical Information and how to request access to related clinical study documents, see here: https:// www.roche.com/research_and_development/ who_we_are_how_we_work/clinical_trials/our_ commitment_to_data_sharing.htm.

\section{ACKNOWLEDGEMENTS}

The authors would like to thank the study participants and their families, as well as the study investigators, research coordinators and nurses. Editorial assistance for the development of this manuscript, under the direction of the authors, was provided by Adele Blair, PhD, and Phoebe Tate, MSc, of Ashfield MedComms, an Ashfield Health company, and funded by F. Hoffmann-La Roche Ltd and Genentech, Inc. The study was funded by F. Hoffmann-La Roche Ltd and Genentech, Inc.

\section{ORCID}

Anisha M. Patel (ID) https://orcid.org/0000-0003-1902-905X Kathleen Poulos (iD) https://orcid.org/0000-0001-5101-0100 Karina Raimundo (iD https://orcid.org/0000-0001-7149-0914 Gabriel Wong (iD) https://orcid.org/0000-0003-2904-7550 Janet Bernard (iD https://orcid.org/0000-0002-4244-0114 Richard H. Ko iD https://orcid.org/0000-0002-7603-6697

\section{REFERENCES}

1. Mannucci PM, Tuddenham EG. The hemophilias--from royal genes to gene therapy. N Engl J Med 2001; 344(23): 1773-9. doi: 10.1056/nejm200106073442307.

2. Srivastava A, Santagostino E, Dougall A, et al. WFH Guidelines for the Management of Hemophilia, 3rd edition. Haemophilia 2020; 26 (Suppl 6): 1-158. doi: 10.1111/hae.14046.

3. Knobe K, Berntorp E. Haemophilia and joint disease: pathophysiology, evaluation, and management. J Comorb 2011; 1: 51-9. doi: 10.15256/joc.2011.1.2.

4. Oldenburg J, Shima M, Kruse-Jarres R, et al. Outcomes in children with hemophilia A with inhibitors: Results from a noninterventional study. Ped Blood Cancer 2020; 67: e28474. doi: 10.1002/pbc.28474.

5. Witkop M, Neff A, Buckner TW, et al. Self-reported prevalence, description and management of pain in adults with haemophilia: methods, demographics and results from the Pain, Functional Impairment, and Quality of life (P-FiQ) study. Haemophilia 2017; 23(4): 556-65. doi: 10.1111/ hae.13214.

6. Manco-Johnson MJ, Lundin B, Funk S, et al. Effect of late prophylaxis in hemophilia on joint status: a randomized trial. J Thromb Haemost 2017; 15(11)2115-24. doi: 10.1111/ jth.13811.

7. Franchini $M$. The modern treatment of haemophilia: a narrative review. Blood Transfus 2013; 11(2): 178-82. doi: 10.2450/2012.0166-11.

8. Lambert T, Benson G, Dolan G, et al. Practical aspects of extended half-life products for the treatment of haemophilia. Ther Adv Hematol 2018; 9(9): 295-308. doi: 10.1177/2040620718796429.

9. Thornburg CD, Duncan NA. Treatment adherence in hemophilia. Patient Prefer Adherence 2017; 11: 1677-86. doi: 10.2147/PPA.S139851.

10. Wight J, Paisley S. The epidemiology of inhibitors in haemophilia A: a systematic review. Haemophilia 2003; 9(4): 418-35. doi: 10.1046/j.1365-2516.2003.00780.x.

11. Shapiro AD, Mitchell IS, Nasr S. The future of bypassing agents for hemophilia with inhibitors in the era of novel agents. J Thromb Haemost 2018; 16(12): 2362-74. doi: 10.1111/ jth.14296.

12. Lacroix-Desmazes S, Voorberg J, Lillicrap D, Scott DW, Pratt KP. Tolerating factor VIII: Recent progress. Front Immunol 2019; 10: 2991. doi: 10.3389/fimmu.2019.02991.

13. Carcao M, Escuriola-Ettingshausen C, Santagostino E, et al. The changing face of immune tolerance induction in haemophilia A with the advent of emicizumab. Haemophilia 2019; 25(4): 676-84. doi: 10.1111/hae.13762.

14. Kitazawa $T$, Igawa $T$, Sampei $Z$, et al. A bispecific antibody to factors IXa and $X$ restores factor VIII hemostatic activity in a hemophilia A model. Nat Med 2012; 18(10): 1570-4. doi: 10.1038/nm.2942.

15. Oldenburg J, Mahlangu JN, Kim B, et al. Emicizumab prophylaxis in hemophilia A with inhibitors. $N$ Engl J Med 2017; 377(9): 809-18. doi: 10.1056/NEJMoa1703068.

16. Mahlangu J, Oldenburg J, Paz-Priel I, et al. Emicizumab prophylaxis in patients who have hemophilia A without inhibitors. N Engl J Med 2018; 379(9): 811-22. doi: 10.1056/ NEJMoa1803550. 
17. Pipe SW, Shima M, Lehle M, et al. Efficacy, safety, and pharmacokinetics of emicizumab prophylaxis given every 4 weeks in people with haemophilia A (HAVEN 4): a multicentre, open-label, non-randomised phase 3 study. Lancet Haematol 2019; 6(6): E295-E305. doi: 10.1016/S2352-3026(19)30054-7.

18. Young $G$, Liesner $R$, Chang $T$, et al. A multicenter, open-label, phase 3 study of emicizumab prophylaxis in children with hemophilia A with inhibitors. Blood 2019; 134(24): 2127-38. doi: 10.1182/blood.2019001869.

19. US Food and Drug Administration. HEMLIBRA ${ }^{\circledR}$ (emicizumabkxwh) injection for subcutaneous use, prescribing information [Initial U.S. approval: 2017] 2018. Available from: https://www gene.com/download/pdf/hemlibra_prescribing.pdf (accessed August 2021).

20. Kitazawa T, Esaki K, Tachibana T, et al. Factor VIIla-mimetic cofactor activity of a bispecific antibody to factors IX/IXa and $X / X a$, emicizumab, depends on its ability to bridge the antigens. Thromb Haemost 2017; 117(7): 1348-57. doi: 10.1160/th17-01-0030

21. Callaghan M, Negrier C, Paz-Priel I, et al. Long-term outcomes with emicizumab prophylaxis for hemophilia A with or without FVIII inhibitors from the HAVEN 1-4 studies. Blood 2021; 137(16): 2231-42. doi: 10.1182/blood.2020009217.

22. Schmitt $\mathrm{C}$, Adamkewicz JI, Xu J, et al. Pharmacokinetics and pharmacodynamics of emicizumab in persons with hemophilia A with factor VIII inhibitors: HAVEN 1 study. Thromb Haemost 2021; 121(3): 351-60. doi: 10.1055/s-0040-1717114.

23. Batsuli G, Zimowski KL, Tickle K, Meeks SL, Sidonio RF, Jr. Immune tolerance induction in paediatric patients with haemophilia A and inhibitors receiving emicizumab prophylaxis Haemophilia 2019; 25(5): 789-96. doi: 10.1111/hae.13819

24. Batsuli G, Greene A, Meeks SL, Sidonio Jr RF. Emicizumab in tolerized patients with hemophilia A with inhibitors: A singleinstitution pediatric cohort assessing inhibitor status. Res Pract Thromb Haemost 2021; 5(2): 342-8. doi: 10.1002/rth2.12475.

25. Oldenburg J, Mahlangu JN, Bujan W, et al. The effect of emicizumab prophylaxis on health-related outcomes in persons with haemophilia A with inhibitors: HAVEN 1 Study. Haemophilia 2019; 25(1): 33-44. doi: 10.1111/hae.13618.

26. Mancuso ME, Mahlangu J, Sidonio Jr R, et al. Health-related quality of life and caregiver burden of emicizumab in children with haemophilia A and factor VIII inhibitors-Results from the HAVEN 2 study. Haemophilia 2020; 26(6): 1009-18. doi: 10.1111/hae.14183.

27. Skinner MW, Negrier C, Paz-Priel I, et al. The effect of emicizumab prophylaxis on long-term, self-reported physical health in persons with haemophilia A without Factor VIII inhibitors in the HAVEN 3 and HAVEN 4 studies. Haemophilia 2021; 27(5): 854-65. doi: 10.1111/hae.14363.

28. Parnes A, Mahlangu, JN., Pipe, SW., Paz-Priel, I., Lehle, M., Trask, PC., Jiménez-Yuster, V. Patient preference for emicizumab versus prior factor therapy in persons with haemophilia A: results from the HAVEN 3 and HAVEN 4 studies. Haemophilia 2021. doi: 10.1111/hae.14421.

29. Jiménez-Yuste $V$, Peyvandi F, Klamroth $R$, et al. Final Analysis of the STASEY Trial: A Single-arm, Multicenter, Open-label, Phase III Clinical Trial Evaluating the Safety and Tolerability of Emicizumab Prophylaxis in Persons with Hemophilia A (PwHA) with Factor (F)VIII Inhibitors [abstract]. Res Pract Thromb Haemost 2021; 5(2). Available from https://abstracts.isth. org/abstract/final-analysis-of-the-stasey-trial-a-single-armmulticenter-open-label-phase-iii-clinical-trial-evaluatingthe-safety-and-tolerability-of-emicizumab-prophylaxis-inpersons-with-hemophilia-a-pwha/ (accessed December 2021).

30. McCary I, Guelcher C, Kuhn J, et al. Real-world use of emicizumab in patients with haemophilia A: Bleeding outcomes and surgical procedures. Haemophilia 2020; 26(4): 631-6. doi: 10.1111/hae.14005

31. Barg AA, Livnat T, Budnik I, et al. Emicizumab treatment and monitoring in a paediatric cohort: real-world data. $\mathrm{Br} \mathrm{J}$ Haematol 2020; 191(2): 282-90. doi: 10.1111/bjh.16964

32. Ebbert PT, Xavier F, Seaman CD, Ragni MV. Emicizumab prophylaxis in patients with haemophilia A with and without inhibitors. Haemophilia 2020; 26: 41-6. doi: 10.1111/hae.13877.

33. Lenting PJ. Laboratory monitoring of hemophilia A treatments: new challenges. Blood Adv 2020; 4(9): 2111-8. doi: 10.1182/ bloodadvances.2019000849.

34. Santagostino E, Oldenburg J, Chang T, et al.. Surgical experience from four phase III studies (HAVEN 1-4) of emicizumab in persons with haemophilia $A(P w H A)$ with or without FVIII inhibitors. ISTH; 2019; Melbourne, Australia. Available from https://www.emicizumabinfo.com/content/ dam/gene/emicizumabinfo/pdf/presentations/isth-2019-oralhaven-surgeries-santagostino.pdf (accessed December 2021).

35. Nuss R, Hedegaard $H$, Riske $B$, et al. Medical care for haemophilia. Haemophilia 1998; 4: 1365-2516. doi: 10.1046/j.1365-2516.1998.00196.x.

36. Royal S, Schramm W, Berntorp E, et al. Quality-of-life differences between prophylactic and on-demand factor replacement therapy in European haemophilia patients. Haemophilia 2002; 8(1): 44-50. doi: 10.1046/j.1365-2516.2002.00581.x

37. Garcia-Dasi M, Aznar JA, Jimenez-Yuste V, et al. Adherence to prophylaxis and quality of life in children and adolescents with severe haemophilia A. Haemophilia 2015; 21(4): 458-64. doi: 10.1111/hae.12618.

38. Patel AM, Corman SL, Chaplin S, Raimundo K, Sidonio RF. Economic impact model of delayed inhibitor development in patients with hemophilia a receiving emicizumab for the prevention of bleeding events. J Med Econ 2019; 22(12): 1328-37. doi: 10.1080/13696998.2019.1669614.

39. Cortesi PA, Castaman G, Trifirò G, et al. Cost-effectiveness and budget impact of emicizumab prophylaxis in haemophilia $A$ patients with inhibitors. Thromb Haemost 2020; 120(02): 216-28. doi: 10.1055/s-0039-3401822.

40. Zhou ZY, Raimundo K, Patel AM, et al. Model of short- and long-term outcomes of emicizumab prophylaxis treatment for persons with hemophilia A. J Manag Care Spec Pharm 2020; 26(9): 1109-20. doi: 10.18553/jmcp.2020.19406.

41. Lin P-C, Liao Y-M, Tsai S-P, Chang T-T. Immune tolerance induction therapy for patients with hemophilia A and FVIII inhibitors particularly using low-dose regimens. Pediatr Blood Cancer 2011; 57: 1029-33. doi: 10.1002/pbc.23291.

42. Institute for Clinical and Economic Review; New England Comparative Effectiveness Public Advisory Council. Emicizumab for hemophilia A with inhibitors: effectiveness and value. Final evidence report. [Boston, Mass.]: ICER: April 16, 2018. Available from https://collections.nlm.nih.gov/catalog/ nlm:nlmuid-101744955-pdf (accessed December 2021).

43. Institute for Clinical and Economic Review; New England Comparative Effectiveness Public Advisory Council. 
Valoctocogene roaparvovec and emicizumab for hemophilia A: effectiveness and value. Final report. [Boston, Mass.]: ICER, November 20; 2020. Available from https://icer. org/wp-content/uploads/2020/10/ICER_Hemophilia-A_FinalReport_112020.pdf (accessed December 2021).

44. Kelley K, CLark B, Brown V, Sitzia J. Good practice in the conduct and reporting of survey research. Int J Qual Health Care 2003; 15(3): 261-6. doi: 10.1093/intghc/mzg03
HOW TO CITE THIS ARTICLE:

Patel AM, Owens WE, Poulos K, Raimundo K, Wong G, Bernard J, Powers J, Ko RH. Haemophilia A management with emicizumab: A survey of haematologists in the United States. J Haem Pract 2021; 8(1): 155-182.

https://doi.org/10.2478/jhp-2021-0017.

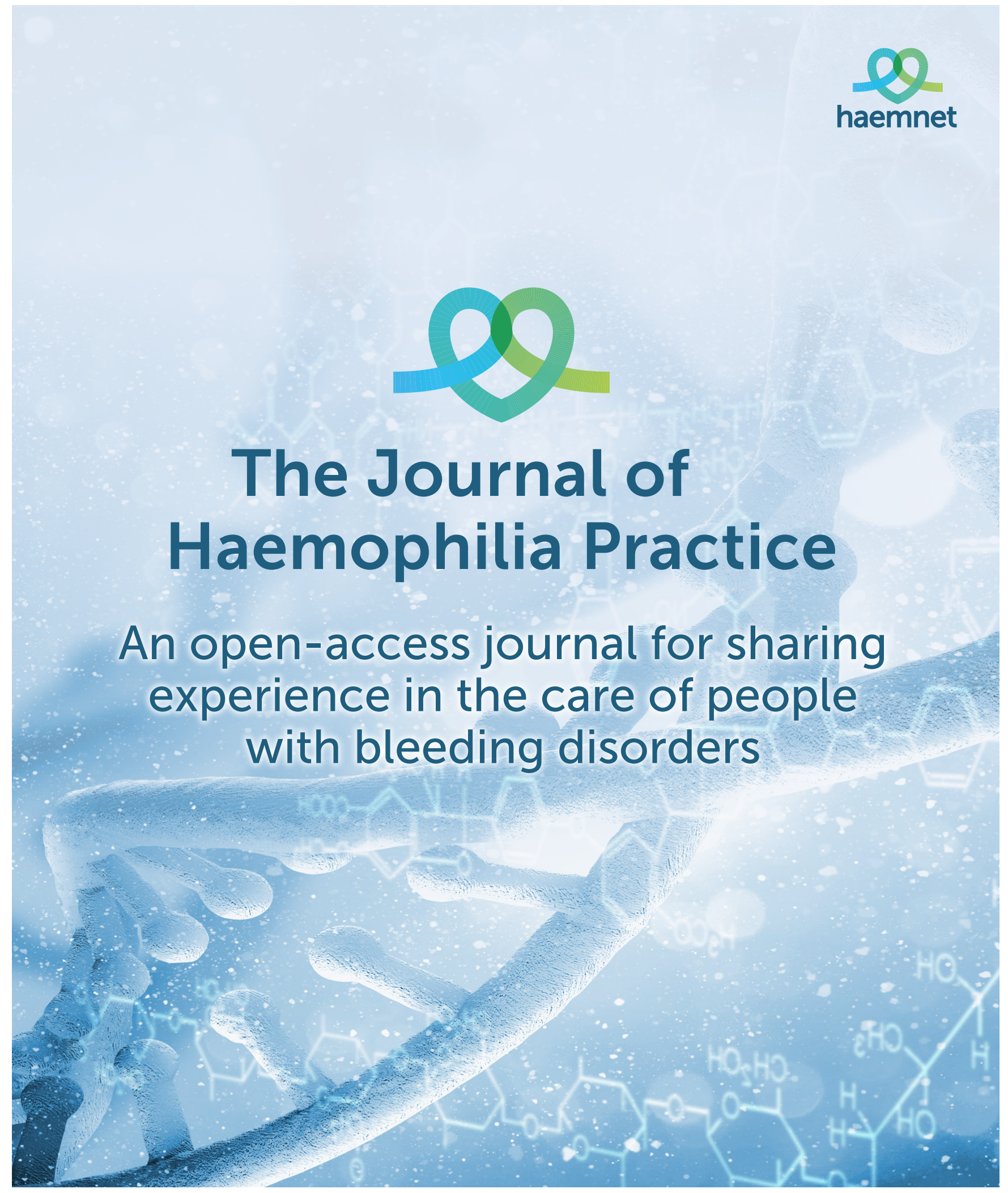




\section{APPENDIX}

Supplementary Table 1. Screening criteria required for survey participation

\begin{tabular}{l|l} 
& REQUIRED CRITERIA \\
\hline Profession & Medical doctor \\
\hline Primary specialty & Haemophilia A \\
\hline Board-certified in haematology? & Yes \\
\hline Number of years of post-residency experience & $\geq 2$ years \\
\hline Geographical location & US \\
\hline Number of PwHArE & \\
PWHA treated in an HTC & $\geq 5$ PWHA \\
PWHA treated in a non-HTC & $\geq 3$ PWHA \\
\hline
\end{tabular}

HTC: haemophilia treatment centre; PwHA: person/people with haemophilia A;

PwHArE: person/people with haemophilia A receiving emicizumab; US: United States

Supplementary Table 2. Geographical location of haematologists

\begin{tabular}{|c|c|}
\hline STATE & NUMBER OF HAEMATOLOGISTS $(\mathrm{N}=50)$ \\
\hline Arizona & 3 \\
\hline California & 6 \\
\hline Connecticut & 1 \\
\hline Florida & 5 \\
\hline Georgia & 1 \\
\hline Illinois & 3 \\
\hline lowa & 1 \\
\hline Kentucky & 2 \\
\hline Louisiana & 3 \\
\hline Michigan & 3 \\
\hline Missouri & 1 \\
\hline Nebraska & 1 \\
\hline New Jersey & 1 \\
\hline New York & 4 \\
\hline North Carolina & 1 \\
\hline Ohio & 3 \\
\hline Pennsylvania & 3 \\
\hline Tennessee & 1 \\
\hline Texas & 2 \\
\hline Virginia & 1 \\
\hline Washington & 2 \\
\hline Wisconsin & 2 \\
\hline
\end{tabular}

It is unknown if any of the surveyed haematologists were from the same haemophilia treatment centre; this was not an exclusion criterion during screening 
SURVEY QUESTIONS (PART 1): INITIATING TREATMENT WITH EMICIZUMAB

FOR QUESTIONS 1-6, WHAT PERCENTAGE OF PWHArE FIT INTO EACH OF THE FOLLOWING CATEGORY RANGES?

\section{PwHArE age (\%)}
a. Infants (0-12 months)
b. Children (1-11 years)
c. Adolescents (12-17 years)
d. Adults (18-65 years)
e. Older adults (66+ years)

2. Previously untreated PWHA (PUPs; not previously treated with FVIII) (\%)

3. Severity of disease (based on FVIII genotype) (\%)

4. Frequency of bleeds for PwHA with mild and moderate disease severity (based on FVIII genotype) while treated with emicizumab [Show if Q3A >0\%; show if Q3B >0\%]

5. Currently have FVIII inhibitors or had FVIII inhibitors that resolved (\%)

6. PwHArE physical activity level (\%)

7. What are the five most important PwHA characteristics that you considered when initiating treatment with emicizumab? Please rank the most important characteristic from the list as number one and the least important as number five [Allow only the top five rankings]

\section{a. Percentage untreated before emicizumab}
a. Mild
b. Moderate
c. Severe

Percentage of those with $\leq 2$ bleeds per year and $>2$ bleeds per year for:
a. Mild
b. Moderate

Percentage of PwHArE that have/or had low-titer inhibitors and that have/or had high-titer inhibitors for:

a. PWHA who currently have FVIII inhibitors
b. PWHA who had FVIII inhibitors that resolved

a. Low impact activities

b. Moderate impact activities

c. High impact activities

d. Typically fit into $>1$ category of physical activity

a. Age

b. Factor level / disease severity

c. Frequency of bleeds

d. Newly diagnosed

e. Previously untreated

f. Safety or efficacy issues with current product

g. Frequency of hospitalisations / emergency room visits

h. Treats prophylactically or not

i. Physical activity level

j. Participation in physically demanding work / school activities

k. PwHA has inhibitors

l. PWHA has high treatment burden (e.g., venous access, frequency of administration)

m. PwHA's distance from emergency treatment

n. PWHA's distance from routine treatment

o. Type of insurance coverage

p. Others (existing health conditions, patient behaviours, caregiver's ability to administer treatment, etc.)
a. I did
b. PwHA (or caregiver) did
c. Other 
SURVEY QUESTIONS (PART 1): INITIATING TREATMENT WITH EMICIZUMAB

FOR QUESTIONS 1-6, WHAT PERCENTAGE OF PWHArE FIT INTO EACH OF THE FOLLOWING CATEGORY RANGES?

9. What are the top five reasons that you advised the PwHArE (or caregiver) or agreed that they switch to / initiate treatment with emicizumab? Please rank the most important reason from the list as number one and the least important as number five

[Allow only the top five rankings] a. PwHA does not adhere to current treatment regimen

b. Venous access issues

c. Efficacy issues with current product

d. Safety issues with current product

e. Need to improve bleed management

f. Cost of treatment to PwHA

g. More effective product available

h. Current treatment product is discontinued

i. Insurance coverage issues

j. PWHA is no longer confident in their treatment

k. PwHA requests emicizumab with no definitive reason

l. Inhibitor development

m. To lower treatment burden (e.g., ease of administration, lower frequency)

n. To improve PwHA quality of life

o. Other

\section{SURVEY QUESTIONS (PART 2): BLEED MANAGEMENT}

10. How do you help PwHArE (or their caregivers) recognise a breakthrough bleed? Please select all that apply. a. We provide educational materials to help $\mathrm{PwHA}$ recognise bleeds

b. PwHA (or caregiver) calls the clinic and describes symptoms and we help diagnose the bleed

c. PwHA (or caregiver) has experience with bleeds and knows when they are having one

d. Other (recommend an ultrasound, etc.)

11. Do you change the guidance that you provide PwHA for treatment of bleeds (with FVIII or BPA) once they start treatment with emicizumab?

12. How has the guidance that you provide PwHA with and without inhibitors for treatment of bleeds (with FVIII or BPAs) changed once they started treatment with emicizumab? a. No [Skip to Q13]

b. Yes
I am less likely to recommend; I recommend about the same; I am more likely to recommend; or not sure/NA, for PwHA with inhibitors and PwHA without inhibitors, for the following categories:

a. Treatment as soon as bleed is suspected

b. Waiting until it is clear they are bleeding, then treat

c. PwHA call an HCP and discuss symptoms and seek help on how to treat the bleed

d. Other treatment guidance

Percentage of PwHA with inhibitors and $\mathrm{PwHA}$ without inhibitors for:

a. Minor bleeds (i.e., requiring a single dose of factor or BPAs to resolve)

b. Significant bleeds (i.e., requiring multiple doses of factor or BPA to resolve) 
14. For each of the following types of bleeds, please select the primary methods for treatment and management of bleeds that you most often suggest to PwHA with inhibitors (or their caregivers) treated with emicizumab. Please select all that apply for each bleed type.
For joint bleeds, muscle bleeds, superficial or other soft tissue bleeds, and other internal bleeds:

a. Wait until it is clear they are bleeding, then treat

b. Treat as soon as they suspect they have a bleed

c. Contact HCP and discuss symptoms and seek help on how to treat the bleed before treatment

d. rFVIla as first-line treatment of bleeds

e. Treat with a single dose of rFVIIa

f. Treat with single dose of activated prothrombin complex concentrates

g. Treat until bleed has been resolved

h. Other

15. For each of the following types of bleeds, please select the primary methods for treatment and management of bleeds that you most often suggest to PwHArE without inhibitors (or their caregiver). Please select all that apply for each bleed type.
For joint bleeds, muscle bleeds, superficial or other soft tissue bleeds, and other internal bleeds:
a. Wait until it is clear they are bleeding, then treat
b. Treat as soon as they suspect they have a bleed
c. Contact HCP and discuss symptoms and seek help on how to treat the bleed before treatment
d. Treat with a single dose of rFVIla
e. Treat until bleed has been resolved
f. Other

\section{SURVEY QUESTIONS (PART 3): MISSED DOSE OR DISCONTINUATION}

16. In general, what would you advise PwHA if they miss a dose of their emicizumab treatment?

17. Which of the following factors would contribute to PwHA discontinuing emicizumab treatment? Please select the top three reasons.

[Accept three answers] a. No guidance

b. Take the next dose as planned

c. Administer as soon as possible and then resume dosing schedule

d. Administer as soon as possible and then contact your healthcare provider regarding resuming dosing schedule

e. Other

a. Issues with insurance

b. Cost of emicizumab

c. Tolerability of subcutaneous injections

d. Tolerability of side effects

e. Compliance with the dosing schedule

f. PwHA choice or desire to discontinue

g. Development of anti-drug antibodies

h. Other

\section{SURVEY QUESTIONS (PART 4): MONITORING}

18. For PwHArE, do you measure FVIII activity more or less frequently than you do for PwHA taking other treatments? a. FVIII activity is measured less frequently in PwHArE

b. FVIII activity is measured at about the same rate in PWHArE

c. FVIII activity is measured more frequently in PWHArE

d. Because I only recently started prescribing emicizumab, FVIII activity is temporarily measured more frequently until I build up more experience

e. Not sure 
19. In general, how often do you measure FVIII activity in your PwHArE with and without inhibitors? Are there any other times that you measure FVIII activity?
For PwHArE with inhibitors and PwHArE without inhibitors, select one of the following for each population:
a. Once per month
b. Once per quarter
c. Once per year
d. Other frequency
e. Not done on a set schedule

For PwHArE with inhibitors and PwHArE without inhibitors, select all that apply for each population:
a. When I feel it is necessary
b. When the patient/caregiver requests it
c. Other reasons
d. Not done at any other times

20. For PwHArE, do you test for FVIII inhibitors more or less frequently than you do for PwHA taking other treatments?

a. PwHArE are tested less frequently

b. PwHArE are tested about the same

c. PwHArE taking emicizumab are tested more frequently

d. Because I only recently started prescribing emicizumab, PwHA are temporarily tested more frequently until build up more experience

e. Not sure

21. How often do you test for FVIII inhibitors in PwHArE with and without inhibitors? one of the following for each population:
For PwHArE with inhibitors and PwHArE without inhibitors, select
a. Once per month
b. Once per quarter
c. Once per year
d. Other frequency
e. Not done on a set schedule

For PwHArE with inhibitors and PwHArE without inhibitors, select all that apply for each population:
a. When I feel it is necessary
b. When the patient/caregiver requests it
c. Other reasons
d. Not done at any other times

\section{SURVEY QUESTIONS (PART 5): ACTIVITY GUIDANCE}

22. Do you provide any specific guidance for recreational physical activities to PwHArE?

23. What type of guidance regarding recreational physical activities do you provide PwHArE? Please select all that apply.
a. No [Skip to Q25]
b. Yes
a. Customised guidance based on their age
b. Customised guidance based on their fitness goals
c. Customised guidance based on severity
d. Avoid activities that have caused bleeds in the past
e. Follow exercise guidelines (e.g., NHF's "Playing it safe")
f. I provide the same guidance to all PwHA regardless of the treatment product they use
g. No specific guidance 
24. Once a PwHA begins treatment with emicizumab, is there any change in the guidance that you provide to PWHArE regarding recreational physical activities? Please select all that apply. a. No change in specific guidance

b. Yes, to avoid activities that have caused bleeds in the past

c. Yes, to gradually increase their activity level

d. Yes, to immediately increase their activity level

e. Yes, to additionally use FVIII before any major physical activity

f. Other

g. No change in specific guidance

h. Yes, to avoid activities that have caused bleeds in the past

i. Yes, to gradually increase their activity level

j. Yes, to immediately increase their activity level

k. Yes, to additionally use FVIII before any major physical activity

l. Other

a. PwHArE are less active

b. PwHArE have similar activity levels

c. PwHArE are more active

d. Not sure

SURVEY QUESTIONS (PART 6): SURGERY MANAGEMENT
26. Have any PwHA had surgery since starting
a. No [go to Q27A] emicizumab?
b. Yes [go to Q27B]

27A. How would you manage PwHArE under the following surgical scenarios? Please select all that apply for each category.

27B. How did you manage PwHArE under the following surgical scenarios? Please select all that apply for each category.

For minor surgery, lower and higher risk of bleeding; major surgery, lower and higher risk of bleeding, select all that apply:

a. Provide close monitoring of bleeding control

b. Manage timing of emicizumab dose

c. Suspend treatment with emicizumab prior to surgery

d. Pre-operative prophylaxis management with other haemostatic treatments (BPA/FVIII) for additional coverage

e. Intra-operative bleed management with other haemostatic treatments (BPA/FVIII)

f. Post-operative bleed management with other haemostatic treatments (BPA/FVIII)

\section{SURVEY QUESTIONS (PART 7): IMMUNE TOLERANCE INDUCTION (ITI)}

28. In PwHArE, do you use ITI with FVIII with the goal of resolving inhibitors?

29A. Which one of the following best describes how you manage PWHArE during ITI?

29B. Which one of the following best describes how you would manage PwHArE during ITI? a. Yes, I use ITI in PwHArE [go to Q29A]

b. No, I haven't used ITI yet, but I probably will in the future [go to Q29B]

c. No, I haven't used ITI yet and I am unlikely to do so in the future [skip to Q34]

a. No additional management of PwHArE during ITI

b. Manage timing of emicizumab dose during ITI

c. Suspend treatment with emicizumab during ITI

d. Other

e. Not sure

[If Q28='a' or 'b', for Q30-33 below, how is/would ITI conducted/be conducted in PwHArE?

Please select all that apply for each]

30. ITI dose for PwHArE, compared with PwHA taking other treatments. a. No change

b. Treat with lower dose for ITI (e.g., standard half-life or extended half-life FVIII 50-100 IU/kg for 2 or 3 times per week)

c. Treat with higher dose for ITI (e.g., standard half-life or extended half-life FVIII >100 IU/kg for $\geq 3$ times per week)

d. Other

e. Not sure 
31. ITI duration in PwHArE, compared with PwHA taking other treatments.
a. No change
b. Attempt ITI for a shorter duration (e.g., $\leq 12$ months)
c. Attempt ITI for a longer duration (e.g., >12 months)
d. Other
e. Not sure

32. Treatment for ITI in PWHArE.

a. Treat with standard half-life FVIII

b. Treat with extended half-life FVIII

c. Treat with plasma-derived FVIII

d. Treat with recombinant FVIII

e. Other

f. Not sure

33. Treatment for bleeds in PWHArE during ITI. a. Treat with standard half-life FVIII (for low-titer inhibitors)

b. Treat with extended half-life FVIII (for low-titer inhibitors)

c. Treat with plasma-derived FVIII (for low-titer inhibitors)

d. Treat with recombinant FVIII (for low-titer inhibitors)

e. Treat with low-dose activated prothrombin complex concentrate

f. Treat with rFVIIa

g. Other

h. Not sure

\section{SURVEY QUESTIONS (PART 8): RESOURCE USE AND CARE}

34. In general, since your $\mathrm{PWHA}$ have started emicizumab, do they seek care at your clinic, outpatient clinic, or ER more or less frequently than before, for each of the following?

35. In general, do your PwHArE seek care at your clinic, outpatient clinic, or ER more or less frequently than your PwHA on other treatments, for each of the following?

36. What are the top five factors that impact the frequency of non-routine care office visits for PwHArE? Please rank the most important factor from the list as number one and the least important as number five [Allow only the top five rankings]

37. For PwHArE do you recommend they keep more or less FVIII/BPA doses at hand for the bleed treatment, than you recommend for $\mathrm{PWHA}$ on other treatments? Note: Assume everything about $\mathrm{PwHA}$ is the same except their treatment.
Since starting emicizumab, PwHArE are seen less frequently, about the same, or more frequently; for each of the following:
a. Routine care (i.e., annual visit, scheduled appointments)
b. Non-routine care (i.e., trauma, major bleed, surgery)
c. Follow-up care (i.e., after a surgery, trauma, etc)
d. Not sure

Compared with PwHA on other HA treatments PwHArE are seen less frequently, about the same, or more frequently; for each of the following:

a. Routine care (i.e., annual visit, scheduled appointments)

b. Non-routine care (i.e., trauma, major bleed, surgery)

c. Follow-up care (i.e., after a surgery, trauma, etc)

d. Not sure

a. Age

b. Factor level

c. Breakthrough bleeds (non-trauma)

d. Bleeds caused by trauma

e. Follow-up after hospitalisations / emergency room visits

f. Need to alter treatment regime or change treatment

g. Need guidance on FVIII or BPA use for bleeds

h. Change in physical activity level

i. Participation in physically demanding work / school activities

j. Inhibitors status

k. Training with self-infusion

l. Change in insurance coverage

m. Other (existing health conditions, patient behaviours, etc.)

a. Less FVIII/BPA product doses at hand

b. Same FVIII/BPA product doses at hand

c. More FVIII/BPA product doses at hand

d. Specific to each PwHA

e. Not sure 
38. For PwHArE, how many BPA or FVIII doses do you typically advise they keep at hand in case of breakthrough bleeding?

39. How does PwHA's level of adherence with emicizumab compare with PwHA taking other treatments? a. 1-2 doses

b. 3-4 doses

c. $>4$ doses

d. Specific to each PWHA

e. Not sure

a. Adherence with emicizumab is significantly worse than with PwHA taking other treatments

b. Adherence with emicizumab is worse than with PwHA taking other treatments

c. Adherence with emicizumab is about the same than with PwHA taking other treatments

d. Adherence with emicizumab is better than with PwHA taking other treatments

e. Adherence with emicizumab is significantly better than with PwHA taking other treatments

f. Not sure
40. How do you monitor PwHA's level of adherence with emicizumab? Select all that apply

a. Do not monitor adherence

b. Periodic phone calls/texts to the PwHA (or caregiver)

c. Try to assess adherence at each visit

d. Work with multidisciplinary care team to track adherence

e. Review treatment log with PwHA to assess adherence

f. Other

41. How do you encourage adherence with emicizumab? Select all that apply. a. We are unable to do anything to encourage or maintain adherence with emicizumab

b. Reminder phone calls/texts to the PwHArE (or caregiver)

c. Discuss the importance of adherence at each visit

d. Provide literature (e.g., tips for adherence or importance of adherence)

e. Provide access to online resources (websites, patient portals, etc.)

f. Recommend educational websites

g. Plan and provide interventions by a healthcare provider for PwHArE who you suspect are not adhering

h. Other

\section{SURVEY QUESTIONS (PART 9): TREATMENT ACCESS}

42. How often do PwHA encounter health insurance coverage issues for the following (never, rarely, occasionally, frequently, always, not sure): a. Coverage for emicizumab

b. Coverage for FVIII/BPA for bleed treatment while taking emicizumab

c. Care for HA while taking emicizumab (i.e. office visits, physical therapy, etc.)

\section{SURVEY QUESTIONS (PART 10): CHANGE IN OVERALL DISEASE MANAGEMENT}

43. How has the level of disease management support that you provide PwHArE changed since they started treatment with emicizumab? a. Less support than PwHA taking other treatments [Skip to Q44]

b. No change, the same support as PwHA taking other treatments [Skip to end]

c. More support than PwHA taking other treatments [Skip to Q45]

d. Not sure [Skip to end] 
44. Why do you provide less disease management support to PwHArE? a. PwHArE have fewer disease-related issues than PwHA taking other treatments

b. PwHArE have a lower disease burden than PwHA taking other treatments

c. PwHArE have fewer bleeds

d. I am not as concerned about PwHArE having breakthrough bleeds

e. I am not as concerned about PwHArE needing emergency care

f. Other [Skip to end]

45. Why do you provide more disease management support to PwHArE? Please select all that apply. a. PwHArE have more disease-related issues than PwHA taking other treatments

b. PwHArE have a greater disease burden than PwHA taking other treatments

c. PwHArE are engaged in more physically demanding activities

d. I am concerned about PwHArE having breakthrough bleeds

e. I am concerned about PwHArE needing emergency care

f. Emicizumab is a newer agent with limited, long-term safety and efficacy data

g. Other

BPA: bypassing agent; ER: emergency room; FVIII: factor VIII; HA: haemophilia A; HCP: healthcare professional; ITI: immune tolerance induction; NA: not applicable; NHF: National Hemophilia Foundation; PwHA: person/people with haemophilia A; PwHArE: person/ people with haemophilia A receiving emicizumab; rFVIla: recombinant factor VIla

Pilot survey development

- Initial screening questions and a 60-minute interview with 5 US-based haematologists

- Transcript analysed and pilot survey drafted

\section{Pilot survey testing}

- Interviews with 2 US-based haematologists to review the pilot survey questions

\section{Survey finalized}

- Responses collected during the pilot used to finalise the 30-minute online survey

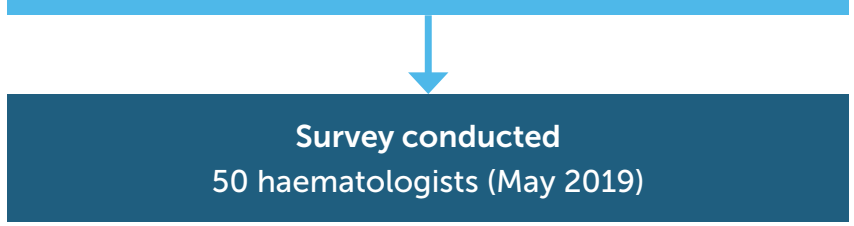

The directory of the Centers for Disease Control and Prevention and the proprietary panel of haematologists at MedPanel were used to identify haematologists to aid with survey development. The five haematologists who participated in the initial screening questions and interviews were different from the two haematologists who reviewed the pilot survey, who again were different from the 50 haematologists who completed the survey. A total of 208 haematologists responded; 83 were screened out due to meeting one or more exclusion criteria and 74 partially completed the screener or survey but did not finish it. One complete survey was excluded, so 50 complete surveys were included in the study. 
Supplementary Figure 2. Primary methods for treatment and management of bleeds most often suggested by the surveyed haematologists in PwHArE with FVIII inhibitors (a) and without inhibitors (b), as reported by haematologists $(\mathrm{N}=50)$

These suggestions are presented as perceived by the haematologists who completed the survey and do not include patient-reported data

2a. PwHArE with FVIII inhibitors

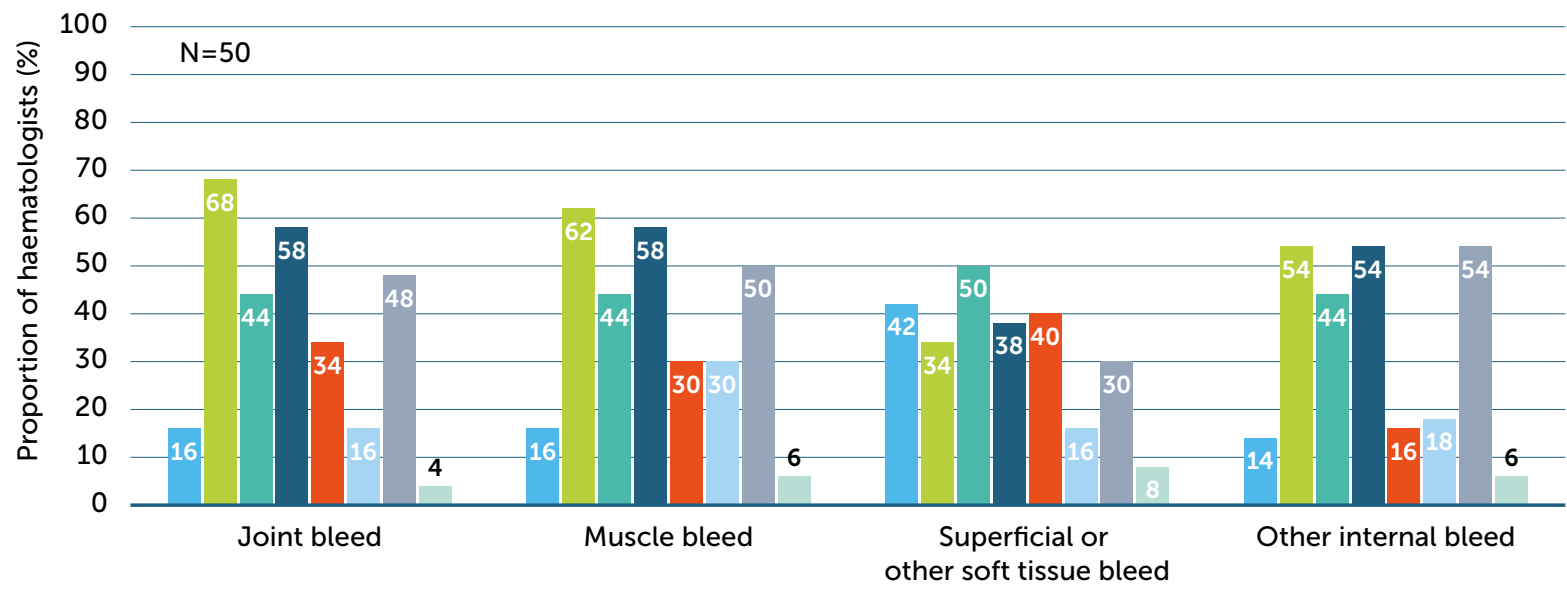

Wait until it is clear a bleed is occurring, then treat

Treat as soon as a bleed is suspected

Contact a HCP for symptom diagnosis and treatment advice before treating the bleed $\quad$ Treat with first-line rFVIla

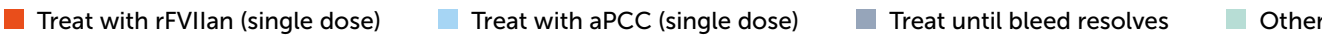

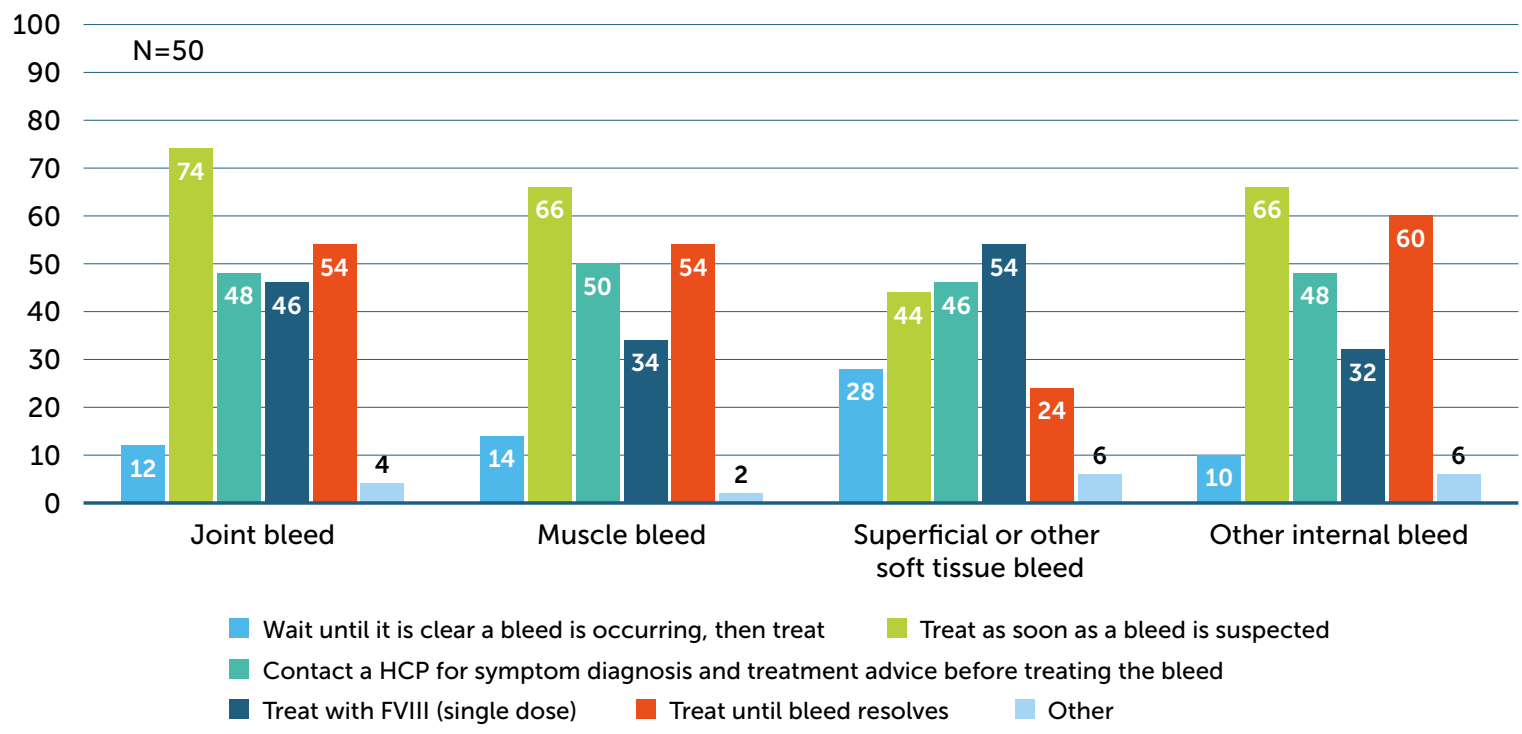

aPCC: activated prothrombin complex concentrate; FVIII: factor VIII; HCP: healthcare professional; PwHArE: person/people with haemophilia A receiving emicizumab; rFVIla: recombinant factor VIla 
Supplementary Figure 3. Proportion of haematologists $(\mathrm{N}=50)$ who $(\mathrm{a})$ recommend PwHArE keep doses of FVIII/BPA on hand versus PwHA receiving other haemophilia A treatments, (b) recommended number of doses that PwHArE should keep on hand, and (c) methods used to encourage adherence to emicizumab prophylaxis

$3 a$.

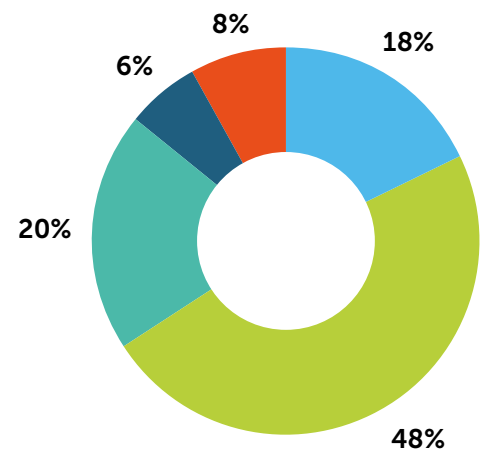

Specific to each individual

Less FVIII/BPA

doses at hand

Not sure 3b.

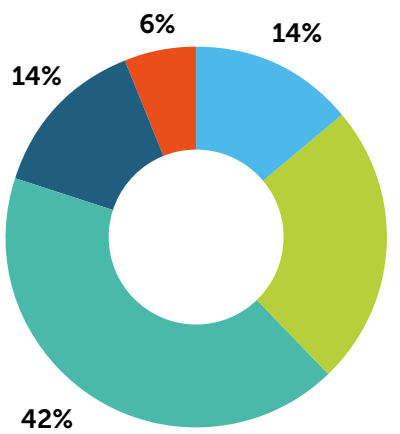

$24 \%$
Specific to each individual
1-2 doses
3-4 doses $\quad$-4 doses
3-4 doses $\quad$ - $>4$ doses

More FVIII/BPA

doses at hand
Not sure

3c.

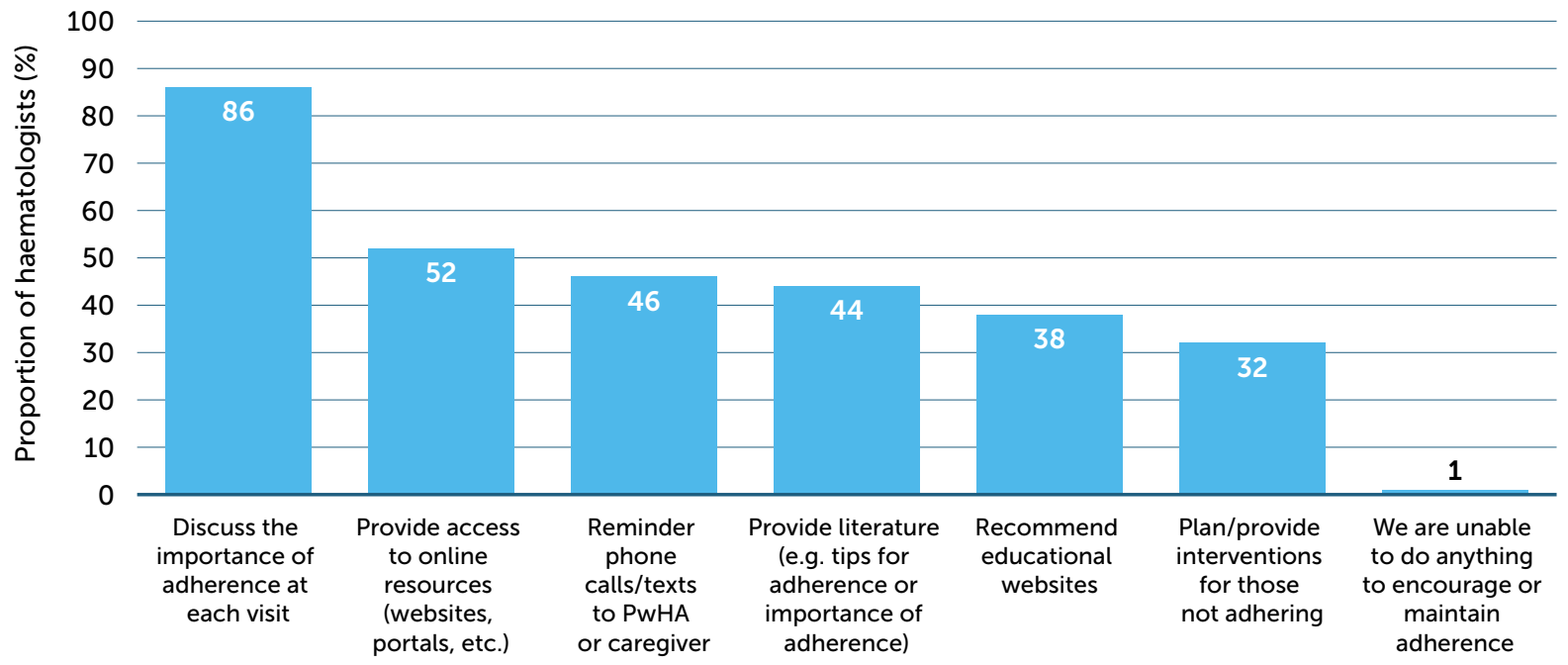

BPA: bypassing agent; FVIII: factor VIII; PwHA: person/people with haemophilia A; PwHArE: person/people with haemophilia $A$ receiving emicizumab 


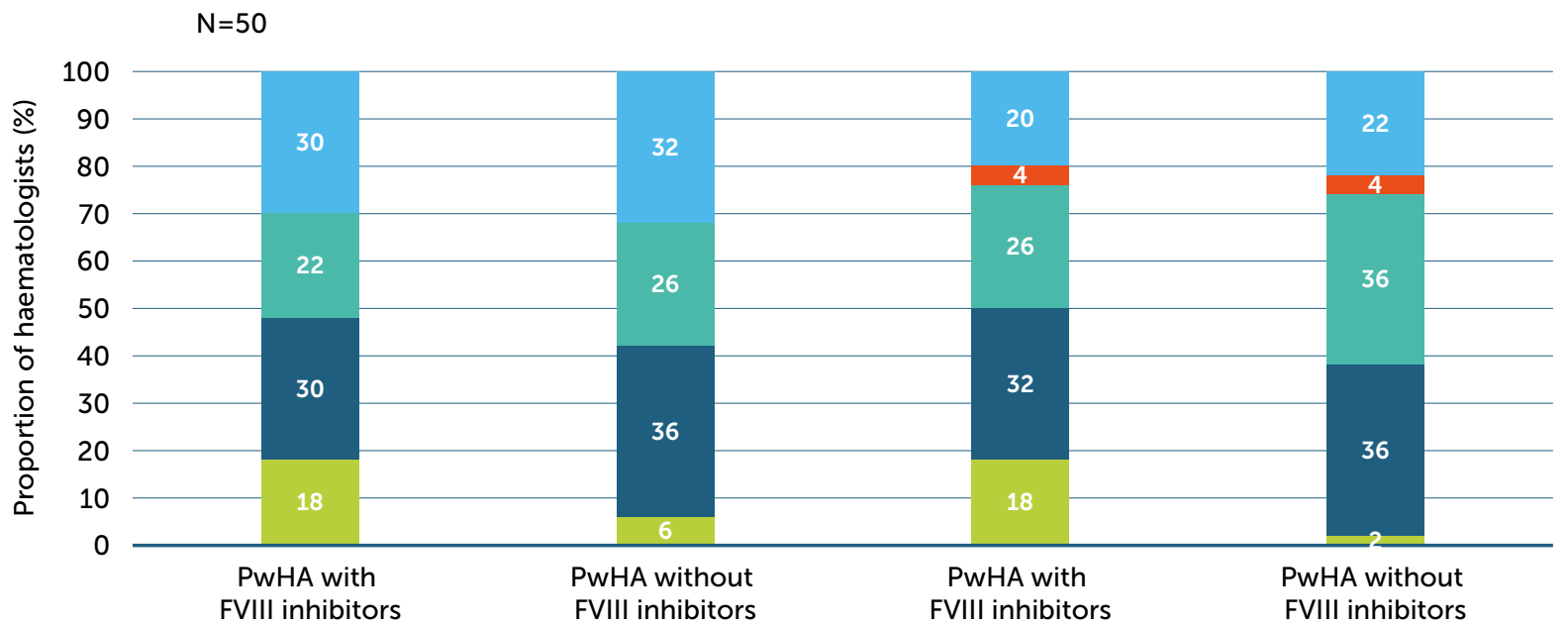

Frequency of measuring FVIII activity

Frequency of testing for FVIII inhibitors

Not done on $\quad$ Other frequency $\quad$ Once per year $\quad$ Once per quarter $\quad$ Once per month
a set schedule

FVIII: factor VIII; PwHA: person/people with haemophilia A; PwHArE: person/people with haemophilia A receiving emicizumab

Supplementary Figure 5. Surgical management strategies adopted by haematologists $(n=11)$ treating PwHA who have had surgery while receiving emicizumab treatment

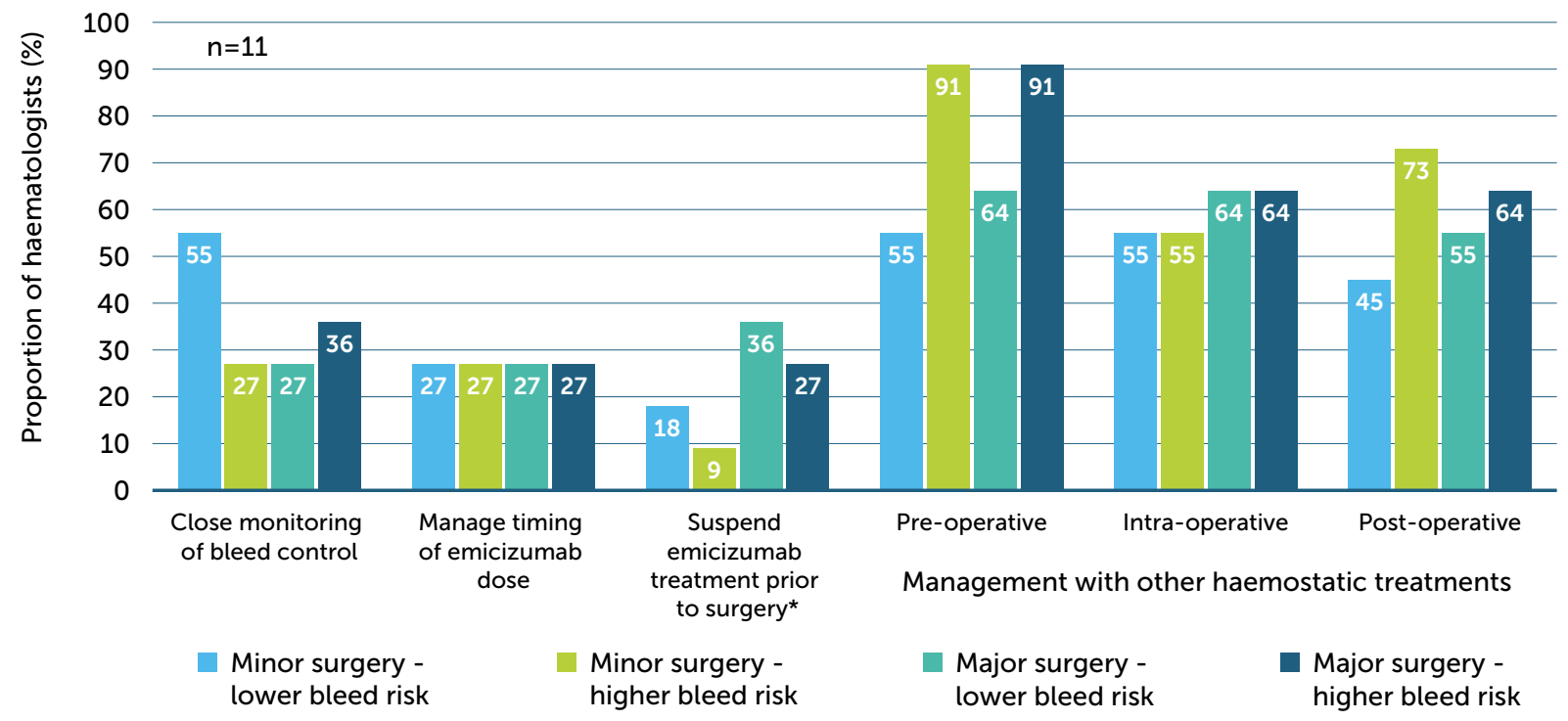

* Due to the long half-life of emicizumab (approximately 30 days), effects of emicizumab can remain for at least 6 months after discontinuation ${ }^{[19]}$

t Other haemostatic treatments include FVIII and BPAs

BPA: bypassing agents; FVIII: factor VIII; PwHA: person/people with haemophilia A 
Supplementary Figure 6. (a) ITI treatment dose and (b) duration in PwHArE compared with PwHA taking other treatments, as reported by haematologists currently using ITI $(n=11)$ and considering using ITI in the future $(n=19)$

$6 a$.

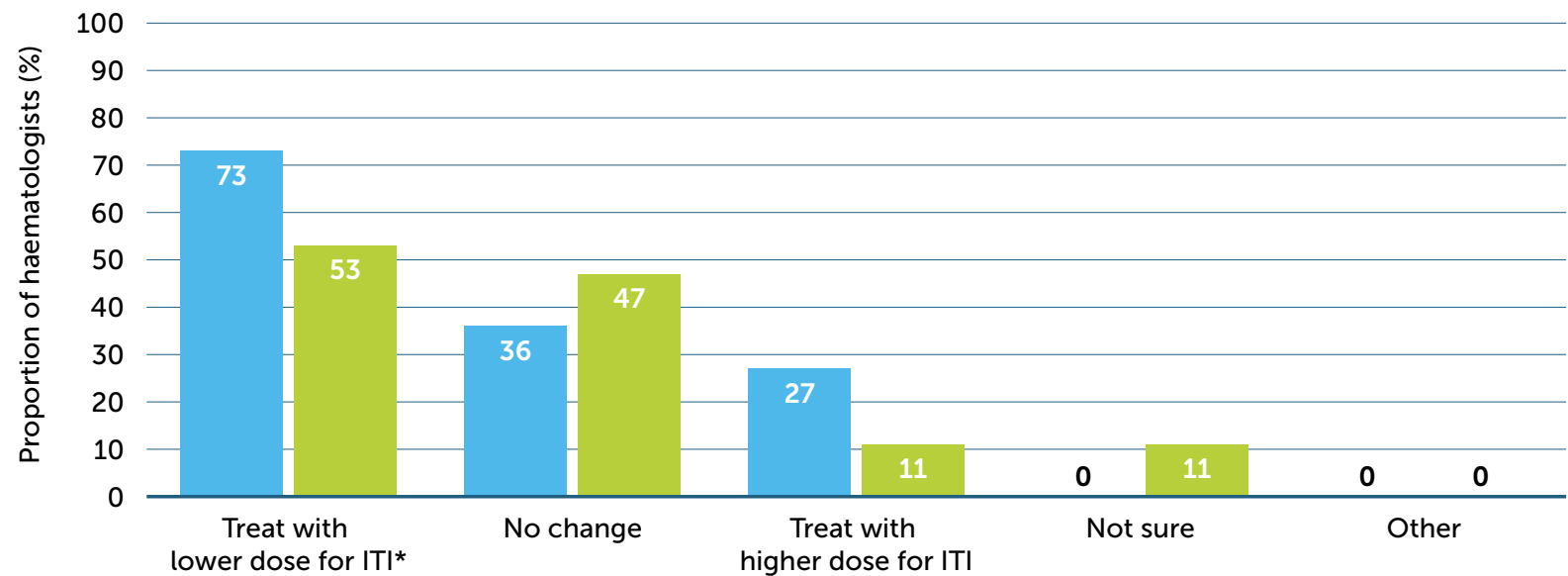

$6 b$.

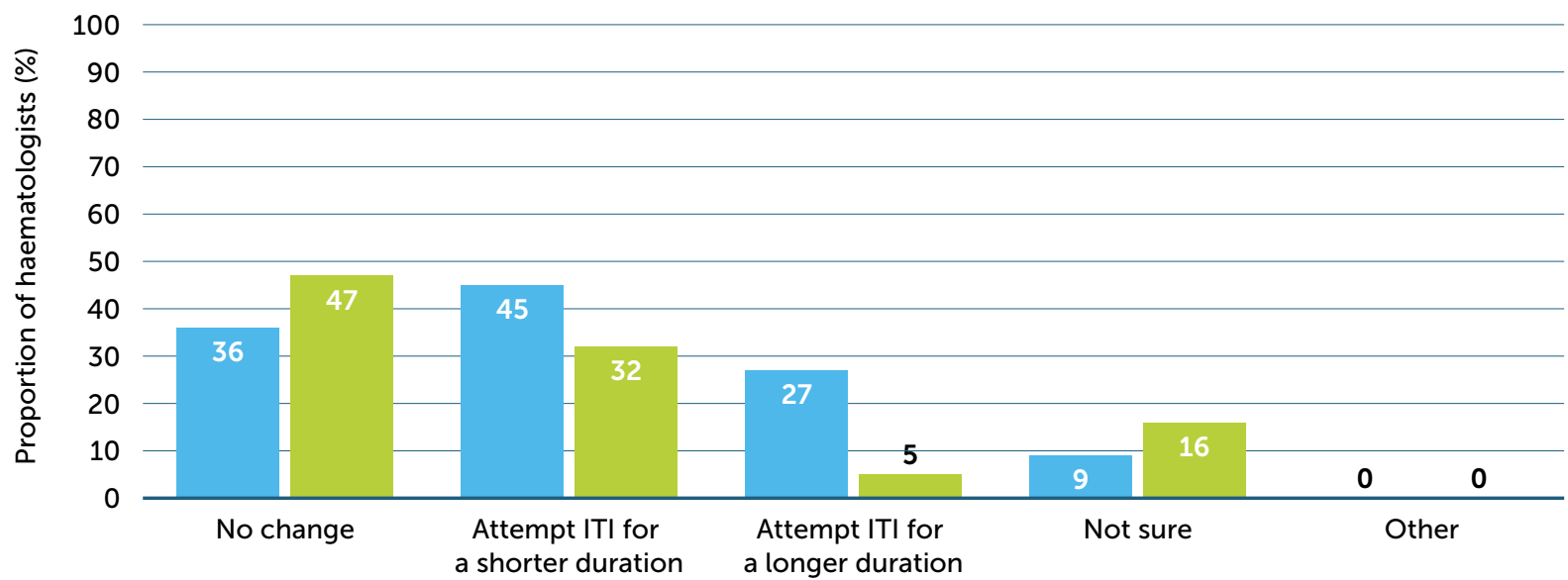

Haematologists currently using ITI $(n=11)$

Haematologists considering using ITI in the future $(n=19)$

FVIII: factor VIII; ITI: immune tolerance induction; PwHA: person/people with haemophilia A; PwHArE: person/people with haemophilia A receiving emicizumab

* Example: standard or extended half-life FVIII (50-100 IU/kg 2 or 3 times per week)

$\dagger$ Example: standard or extended half-life FVIII ( $>100 \mathrm{IU} / \mathrm{kg}>3$ times per week)

‡ Example: $\leq 12$ months

Ij Example: $>12$ months

Supplementary Figure 7. Change in level of disease management support provided to PwHArE compared with PwHA taking other haemophilia $A$ treatments, as reported by haematologists $(\mathrm{N}=50)$

These outcomes are presented as perceived by the haematologists who completed the survey and do not include patient-reported data

PwHA: person/people with haemophilia A; PwHArE: person/ people with haemophilia receiving emicizumab

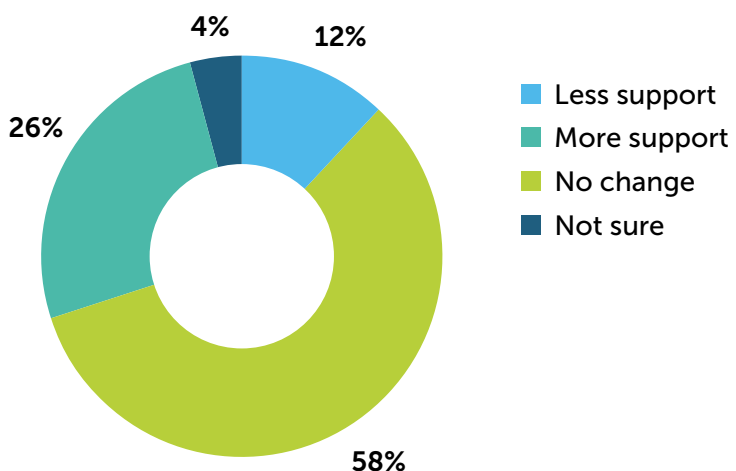

